\title{
The Collapsing Defuzzifier for Discretised Generalised Type-2 Fuzzy Sets
}

\author{
Sarah Greenfield ${ }^{\mathrm{a}, *}$, Francisco Chiclana ${ }^{\mathrm{a}, \mathrm{b}}$ \\ ${ }^{a}$ Institute of Artificial Intelligence, School of Computer Science and Informatics, \\ De Montfort University, Leicester LE1 9BH, UK. \\ ${ }^{b}$ Dept. of Computer Science and Artificial Intelligence, University of Granada, Granada, Spain.
}

\begin{abstract}
The Greenfield-Chiclana Collapsing Defuzzifier is an established efficient accurate technique for the defuzzification of the interval type-2 fuzzy set. This paper reports on the extension of the Collapsing Defuzzifier to the generalised type-2 fuzzy set. Existing techniques for the defuzzification of generalised type-2 fuzzy sets are presented after which the interval Collapsing Defuzzifier is summarised. The collapsing technique is then extended to generalised type-2 fuzzy sets, giving the Generalised GreenfieldChiclana Collapsing Defuzzifier. This is contrasted experimentally with both the benchmark Exhaustive Defuzzifier and the $\alpha$-Planes/Karnik-Mendel Iterative Procedure approach in relation to efficiency and accuracy. The GGCCD is demonstrated to be many times faster than the Exhaustive Defuzzifier and its accuracy is shown to be excellent. In relation to the $\alpha$-Planes/Karnik-Mendel Iterative Procedure approach it is shown to be comparable in accuracy, but faster.
\end{abstract}

Keywords: generalised type-2 fuzzy set, defuzzification, type-reduction, Greenfield-Chiclana Collapsing Defuzzifier, Generalised Greenfield-Chiclana Collapsing Defuzzifier, Representative Embedded Set

\section{Introduction}

Uncertainty is ineradicably present in the factors upon which decisions are made, whether by humans, computers, or a combination of both. In the computational intelligence methodology of fuzzy logic, the ability to deal with uncertainty is desirable as better uncertainty handling engenders more accurate outputs. Type- 2 fuzzy sets are an extension of the original type-1 fuzzy sets in which the sets' membership grades are themselves type-1 fuzzy sets; their main strength is their ability to deal with the second-order uncertainties that arise from multiple sources [26]. The concept dates back to Zadeh's seminal paper of 1975 [54]. They take two forms, the interval, for which every Secondary Membership Grade (SMG) is 1 , and the generalised, where the secondary membership grade may take any value between 0 and 1 . The interval type-2 fuzzy set may be thought of as a blurred version of the type- 1 fuzzy set, and has an inbuilt facility to handle uncertain inputs. However the generalised type- 2 fuzzy set, an augmentation of the interval type-2 fuzzy set, provides uncertainty handling that is subtle and sophisticated [19, 35], owing to its crucial variability of the third dimension [40]. The capability of the type- 2 paradigm to handle uncertainty is explored in $[12,19]$.

The advantage that interval type-2 fuzzy logic has over generalised type-2 fuzzy logic for the application developer, is that it is markedly computationally simpler. For the interval type-2 Fuzzy Inferencing

\footnotetext{
*Corresponding author

Email addresses: s.greenfieldedmu.ac.uk (Sarah Greenfield), chiclana@dmu.ac.uk (Francisco Chiclana)
} 
System (FIS) [40] applications in areas such as control, simulation and optimisation have been developed [2-5, 7-10, 28, 36, 44, 47, 52]; they offer a more sophisticated model of uncertainty than their type-1 counterparts [30], whilst lacking the computational complexity of the generalised type-2 fuzzy set $[24,40]$. Since the turn of the millennium algorithms based on the Karnik-Mendel Iterative Procedure (KMIP) [25, 51] have become the established interval defuzzification techniques [3, 28, 36, 44, 47, 52].

Generalised type-2 fuzzy applications are so far relatively few in number $[23,24,31,35,40,43,46$, 48]. Historically this is in part attributable to the enormous computational cost of generalised type-2 fuzzy inferencing; the recent advances of Vertical Slice Centroid Type-Reduction [34] and the $\alpha$-Planes Method (Subsection 2.3.3) have dramatically reduced the computational cost. Another factor hindering the development of generalised type-2 applications is the fact that designing the secondary membership functions is non trivial. Since the generalised type-2 fuzzy set's facility for dealing with uncertainty is remarkable, it is a shame that it has not as yet been fully exploited. In [23] generalised type-2 fuzzy inferencing has been shown to be superior to interval in classifying facial expressions for emotion recognition. Sanchez, Castillo and Castro demonstrate in [46] that a generalized type-2 fuzzy control system can outperform type-1 and interval type- 2 control systems when external perturbations are present.

The focus of this paper is the discretised Mamdani FIS (Figure 1), in which a crisp numerical input passes through three stages of processing: fuzzification, inferencing, and lastly, the crucial stage of defuzzification. Through defuzzification, the aggregated set produced during the inferencing stage is converted into a crisp number which is the output of the FIS. For discretised type- 1 fuzzy sets, defuzzification is a simple procedure, with several defuzzification techniques available including the centroid, centre of maxima and mean of maxima [29]. In contrast, defuzzification of a discretised type-2 fuzzy set is a process consisting of two stages [38]:

1. Type-reduction, which converts the type-2 fuzzy set to a type-1 fuzzy set known as the TypeReduced Set (TRS);

2. Defuzzification of the TRS.

Owing to its enormous computational complexity, the additional stage of type-reduction in the form of Exhaustive Defuzzification (Subsection 2.2) came to be regarded as a bottleneck [24]. However, in the past decade several alternative type-reduction techniques with good accuracy have been developed, for both the generalised case [15] and the interval case $[16,39,50]$. This paper presents another strategy for generalised type-reduction.

\subsection{Preliminaries}

\subsubsection{Assumptions}

1. All secondary membership functions are convex.

2. The type-2 fuzzy set is contained within a unit cube and may be viewed as a surface represented by $(x, u, z)$ co-ordinates.

3. The centroid method of defuzzification for type-1 fuzzy sets is used.

4. The minimum t-norm is employed.

5. The Grid Method of Discretisation for generalised type-2 fuzzy sets [22] is employed. 


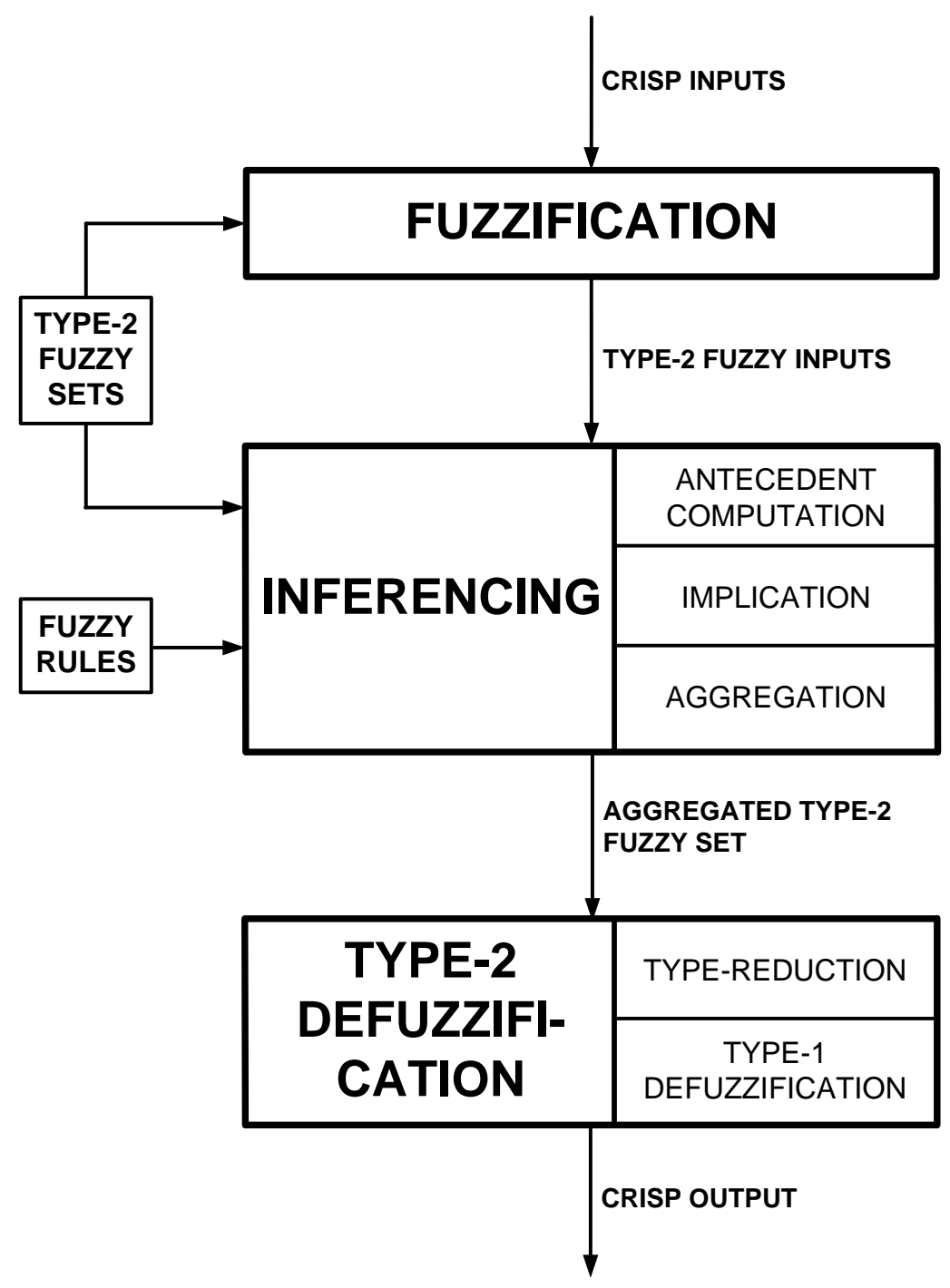

Figure 1: The Mamdani Type-2 FIS [17]. 


\subsubsection{Definitions}

Let $X$ be a universe of discourse. A type- 1 fuzzy set $A$ on $X$ is characterised by a membership function $\mu_{A}: X \rightarrow U(U=[0,1])$ and can be expressed as follows [53]:

$$
A=\left\{\left(x, \mu_{A}(x)\right) \mid x \in X ; \mu_{A}(x) \in U\right\} .
$$

The scalar cardinality of fuzzy set $A$ is the summation of the membership grades of all the elements of $X$ in $A$ [20]: $\|A\|=\sum_{x \in X} \mu_{A}(x)$.

The assumption that the type-2 fuzzy set is contained within a unit cube means that it may be viewed as a surface represented by $(x, u, z)$ co-ordinates with $X \equiv U$. In accordance with [1, 42], a type-2 fuzzy set is defined as

$$
\tilde{A}=\left\{\left(x, u, \mu_{\tilde{A}}(x, u)\right) \mid x \in X ; u \in U ; \mu_{\tilde{A}}(x, u) \in U\right\},
$$

where $\mu_{\tilde{A}}: X \times U \rightarrow U$ is the membership function of $\tilde{A}$. The set $J_{x}=\left\{(x, u) \mid \mu_{\tilde{A}}(x, u)>0\right\}$ is known as the primary membership of $x$, while $\mu_{\tilde{A}}(x, u)$ is known as a secondary membership grade of $x$. For an interval type-2 fuzzy set, Equation (2) reduces to:

$$
\tilde{A}=\{(x,, u, 1)) \mid x \in X ; u \in U\} .
$$

Notice that the assumption of convexity of secondary membership functions implies that $\forall x \in X$ and for any $\left(x, u_{1}\right),\left(x, u_{2}\right) \in J_{x}$ and any $\lambda \in[0,1]$,

$$
\mu_{\tilde{A}}\left[x, \lambda u_{1}+(1-\lambda) u_{2}\right] \geq \lambda \mu_{\tilde{A}}\left(x, u_{1}\right)+(1-\lambda) \mu_{\tilde{A}}\left(x, u_{2}\right) .
$$

Thus, we are assuming that $\mu_{\tilde{A}}(x, u)$ is continuous on the second variable $(u)$ when the first variable $(x)$ is fixed and, as a consequence, $J_{x}$ is a closed interval in $[0,1]$. In this case, we can write $J_{x}=\left[\mu_{\tilde{A}}(x), \bar{\mu}_{\tilde{A}}(x)\right]$, where

$$
\underline{\mu}_{\tilde{A}}(x)=\inf \left\{u \mid(x, u) \in J_{x}\right\} ; \quad \bar{\mu}_{\tilde{A}}(x)=\sup \left\{u \mid(x, u) \in J_{x}\right\},
$$

are known as the the lower and upper membership functions of type- 2 fuzzy set $\tilde{A}$. In the case when $X$ is an interval (as we are assuming that $X \equiv U$ ), the Footprint Of Uncertainty of a type-2 fuzzy set $\tilde{A}$ is the closed region $[0,1]^{2}$ with boundaries the lower and upper membership functions of $\tilde{A}$.

A vertical slice of a type-2 fuzzy set is a plane through the $x$-axis, parallel to the $u-z$ plane $[18,40]$. The degree of discretisation is the separation of the slices [18].

The next section concerns existing defuzzification techniques for type-2 fuzzy sets. Following this, in Section 3 the interval Greenfield-Chiclana Collapsing Defuzzifier is summarised. In Section 4 this method is extended to generalised type-2 fuzzy sets, resulting in the Generalised Greenfield-Chiclana Collapsing Defuzzifier (GGCCD); Section 5 is devoted to its experimental evaluation. Lastly, Section 6 concludes the paper.

\section{Type-Reduction of the Type-2 Fuzzy Set}

\subsection{The Wavy-Slice Representation Theorem}

Associated with type-reduction is the concept of an embedded type-2 fuzzy set (embedded set) or wavy-slice [15, 40] (Figure 2). An embedded set is a special kind of type-2 fuzzy set, which relates to the type-2 fuzzy set in which it is embedded in this way: For every primary domain value, $x$, there is a unique secondary domain value, $u$, plus the associated secondary membership grade that is determined by the primary and secondary domain values, $\mu_{\tilde{A}}(x, u)$. 


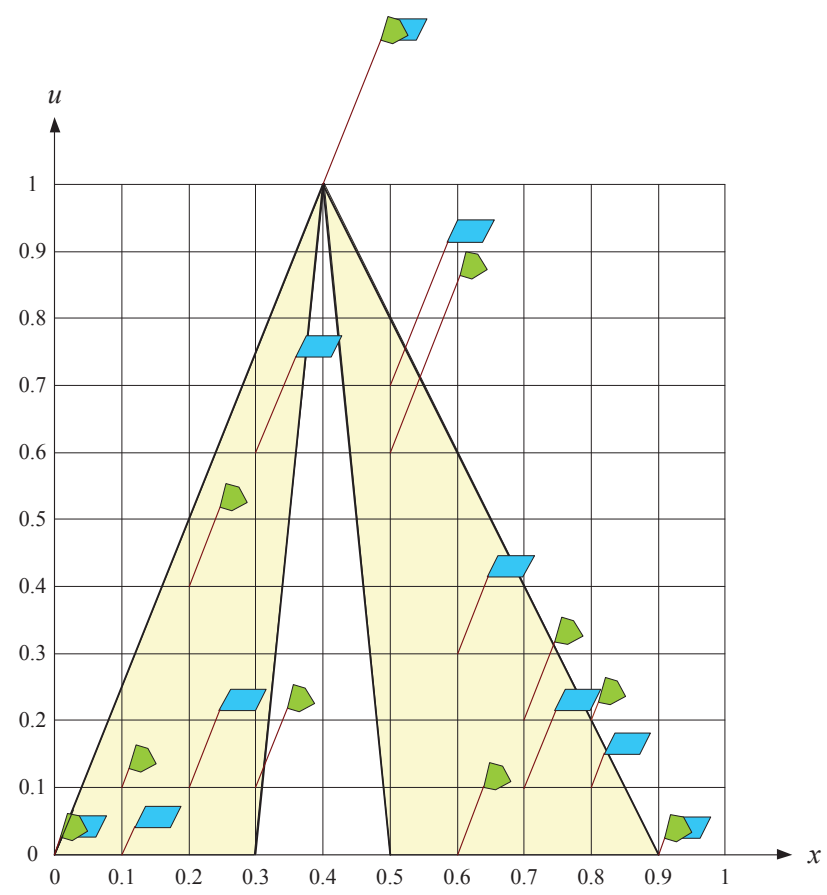

Figure 2: Two embedded type-2 fuzzy sets, indicated by different flag styles. The flag height indicates the secondary membership grade. The degree of discretisation of the primary and secondary domains is 0.1 . The FOU is the shaded region. (Figure adapted from [40].)

Definition 1 (Embedded Set [18]). “Let Ã be a type-2 fuzzy set in X. For discrete universes of discourse $X$ and $U$, an embedded type- 2 set $\tilde{A}_{e}$ of $\tilde{A}$ is defined as the following type-2 fuzzy set

$$
\tilde{A}_{e}=\left\{\left(x_{i}, u_{i}, \mu_{\tilde{A}}\left(x_{i}, u_{i}\right)\right) \mid \forall i \in\{1, \ldots, N\}: x_{i} \in X u_{i} \in U\right\} \text {. }
$$

$\tilde{A}_{e}$ contains exactly one element from $J_{x_{1}}, J_{x_{2}}, \ldots, J_{x_{N}}$, namely $u_{1}, u_{2}, \ldots, u_{N}$, each with its associated secondary grade, namely $\mu_{\tilde{A}}\left(x_{1}, u_{1}\right), \mu_{\tilde{A}}\left(x_{2}, u_{2}\right), \ldots, \mu_{\tilde{A}}\left(x_{N}, u_{N}\right) . "$

Mendel and John have demonstrated that a type-2 fuzzy set is definable as the union of its embedded type-2 fuzzy sets [40]. This powerful result is known as the type-2 fuzzy set Representation Theorem or Wavy-Slice Representation Theorem. The Wavy-Slice Representation Theorem is formally stated thus [40, page 121]:

"Let $\tilde{A}_{e}^{j}$ denote the $j$ th embedded type-2 fuzzy set for type-2 fuzzy set $\tilde{A}$, i.e.,

$$
\tilde{A}_{e}^{j} \equiv\left\{\left(x_{i}, u_{i}^{j}, \mu_{\tilde{A}}\left(x_{i}, u_{i}^{j}\right)\right), i=1, \ldots, N\right\}
$$

where $u_{i}^{j} \in J_{x_{i}}, i=1, \ldots, n$. Then $\tilde{A}$ may be represented as the union of its embedded type-2 fuzzy sets, i.e.,

$$
\tilde{A}=\sum_{j=1}^{n} \tilde{A}_{e}^{j}, \text { where } n \equiv \prod_{i=1}^{N} M_{i}, M_{i} \text { being the cardinality of } J_{x_{i}} .
$$




\subsection{Exhaustive Defuzzification}

The strategy known as Exhaustive Defuzzification, so called because every embedded set is processed in turn, is built upon the foundation of the wavy slice Representation Theorem and is therefore precise ${ }^{1}$ [40]. However it is a very inefficient method owing to its high computational complexity deriving from the large number of embedded sets each needing to be processed. Its first and main stage consists of type-reduction of the type-2 fuzzy set to form the TRS [15], defined thus:

Definition 2 (TRS of a Type-2 Fuzzy Set [14]). “The TRS associated with a type-2 fuzzy set A with primary domain $X$ discretised into $N$ points $X=\left\{x_{1}, x_{2}, \ldots, x_{N}\right\}$, is

$$
C_{\tilde{A}}=\left\{\left(\frac{\sum_{i=1}^{N} x_{i} \cdot u_{k_{i}}}{\sum_{i=1}^{N} u_{k_{i}}}, \mu_{\tilde{A}}\left(x_{1}, u_{k_{1}}\right) * \ldots * \mu_{\tilde{A}}\left(x_{N}, u_{k_{N}}\right)\right) \mid \forall\left(u_{k_{1}}, u_{k_{2}}, \ldots, u_{k_{N}}\right) \in U^{N}\right\},
$$

where * is a t-norm."

Embedded sets (Figure 2) are referred to implicitly in (5) and explicitly in Algorithm 1. For the TRS

\section{Input: a discretised generalised type-2 fuzzy set \\ Output: a discrete type-1 fuzzy set (the TRS)}

\section{1 forall embedded sets do}

2 find the minimum secondary membership grade $(z)$;

3 calculate the primary domain value $(x)$ of the type-1 centroid of the embedded type-2 fuzzy set ;

$4 \quad$ pair the secondary grade $(z)$ with the primary domain value $(x)$ to give set of ordered pairs $(x, z)\{x$-values may correspond to multiple $z$-values $\}$;

5 end

6 forall primary domain $(x)$ values do

7 select the maximum secondary grade $\{$ make each $x$ correspond to a unique value $\}$; 8 end

Algorithm 1: Exhaustive type-reduction of a discretised type-2 fuzzy set to a type-1 fuzzy set, adapted from Mendel [38].

of an interval type-2 fuzzy set, Definition 2 reduces to:

Definition 3 (TRS of an Interval Type-2 Set [16]). "The TRS associated with an interval type-2 fuzzy set $\tilde{A}$ with primary domain $X$ discretised into $N$ points $X=\left\{x_{1}, x_{2}, \ldots, x_{N}\right\}$, is

$$
C_{\tilde{A}}=\left\{\left(\frac{\sum_{i=1}^{N} x_{i} \cdot u_{k_{i}}}{\sum_{i=1}^{N} u_{k_{i}}}, 1\right) \mid \forall\left(u_{k_{1}}, u_{k_{2}}, \ldots, u_{k_{N}}\right) \in U^{N}\right\} .
$$

\subsection{Existing Alternatives to Exhaustive Defuzzification}

Strategies have been developed that reduce the computational complexity of generalised type- 2 defuzzification [22, 32, 34, 55]. In [15] three notable strategies were evaluated, the Sampling Method, Vertical Slice Centroid Type-Reduction, and the $\alpha$-Planes Method:

\footnotetext{
${ }^{1}$ Discretisation in itself brings an unavoidable element of approximation. However the Exhaustive Method does not subsequently introduce further inaccuracies.
} 


\subsubsection{The Sampling Defuzzifier}

The Sampling Method of Defuzzification [22] is an efficient, cut-down alternative to defuzzification via the Exhaustive Method. By processing only a relatively small sample of embedded sets (Definition 1), the computational complexity of type-reduction is drastically reduced. A full exposition of this technique is to be found in [22]. This is a non-deterministic strategy in which the number of embedded sets sampled is a parameter decided by the application developer.

\subsubsection{Vertical Slice Centroid Type-Reduction}

Lucas et al.'s 2007 proposal of Vertical Slice Centroid Type-Reduction (VSCTR) [34] is straightforward and highly intuitive. In this approach the type-2 fuzzy set is cut into vertical slices, each of which is defuzzified as a type-1 fuzzy set. By pairing the domain value with the defuzzified value of the vertical slice, a type-1 fuzzy set is formed, which is easily defuzzified to give the defuzzified value of the type-2 fuzzy set. Though chronologically preceding it, this method is a generalisation of the Nie-Tan Method for interval type-2 fuzzy sets [45].

\subsubsection{The $\alpha$-Plane Representation}

In 2008 Liu [32, 41] proposed the $\alpha$-Planes Representation ${ }^{2}$. The defuzzification technique based on the $\alpha$-Planes Representation was originally conceived as a generalisation of the KMIP. By this technique a generalised type- 2 fuzzy set is decomposed into a set of $\alpha$-planes, which are horizontal slices akin to interval type-2 fuzzy sets. By repeated application of an interval defuzzification method, Liu [32] has shown that a generalised type-2 fuzzy set may be type-reduced ${ }^{3}$. According to Melin et al. [37] "... $\alpha$ planes both enable the representation of, and computation with, general type-2 fuzzy sets." The growing number of generalised type- 2 fuzzy applications are largely attributable to the $\alpha$-planes/zSlices method and include $[6,11,27,33]$.

\section{The Greenfield-Chiclana Collapsing Defuzzifier}

For the interval type-2 fuzzy set, a computationally simple alternative to the Exhaustive Method is the Greenfield-Chiclana Collapsing Defuzzifier (GCCD) [20]. From the outset envisaged as a stepping stone to generalised defuzzification, the GCCD converts an interval type-2 fuzzy set into a type-1 fuzzy set which approximates to the Representative Embedded Set (RES), whose defuzzified value is by definition equal to that of the original type-2 set (Figure 3). We term this type-1 set the Representative Embedded Set Approximation (RESA). As a type-1 set, the RESA may then be defuzzified straightforwardly. Hence the collapsing process reduces the computational complexity of type-2 defuzzification.

Full details of the collapsing algorithm may be found at [20]. We formally state the Simple ${ }^{4}$ Representative Embedded Set Approximation:

Theorem 1 (Simple Representative Embedded Set Approximation [20]). "The membership function of the embedded set $R$ derived by dynamically collapsing slices of a discretised type-2 interval fuzzy set $\tilde{A}$,

\footnotetext{
${ }^{2}$ Independently to Liu, and at about the same time, Wagner and Hagras introduced the notion of zSlices [49], a concept equivalent to that of $\alpha$-Planes.

${ }^{3}$ Indeed this technique generalises any interval method [13] including the Greenfield-Chiclana Collapsing Defuzzifier $[13,20]$, or the Nie-Tan Method [45]. However, in this paper we generalise the interval collapsing method without recourse to the $\alpha$-planes decomposition.

${ }^{4}$ In [20], we used the term 'simple' to describe an interval type-2 fuzzy set in which each vertical slice consists of only two points, corresponding to $L$ and $U$. The term is redundant in the context of this paper.
} 


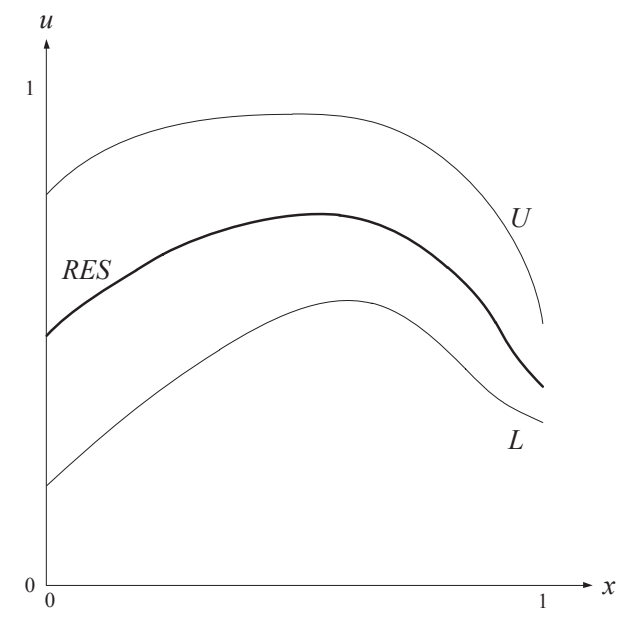

Figure 3: A Representative Embedded Set (continuous case).

having lower membership function $L$, and upper membership function $U$, is:

$$
\mu_{R}\left(x_{i}\right)=\mu_{L}\left(x_{i}\right)+r_{i}
$$

with

$$
r_{i}=\frac{\left(\|L\|+\sum_{j=1}^{i-1} r_{j}\right) b_{i}}{2\left(\|L\|+\sum_{j=1}^{i-1} r_{j}\right)+b_{i}},
$$

and $b_{i}=\mu_{U}\left(x_{i}\right)-\mu_{L}\left(x_{i}\right), r_{0}=0$."

This is an iterative formula. Collapsing proceeds vertical slice by vertical slice. The first slice is collapsed, the first $u$-value of the RESA calculated, the next slice is collapsed and the second $u$-value of the RESA calculated, and so on until all the slices have been collapsed. In this formula $b_{i}$ is the blur for vertical slice $i$, i.e. the difference between the upper membership function and the lower membership function for slice $i . r_{i}$ is the amount by which the $u$-value of $L$ must be increased to give the $u$-value of the RESA $R$.

There are many variants of the collapsing strategy, since slice collapse may proceed in any slice order. The different variants give rise to slightly different defuzzified values [21]. It has been demonstrated practically and theoretically that the two-pass Collapsing Outward Right-Left (CORL) is the most accurate variant [21].

\section{The Generalised Greenfield-Chiclana Collapsing Defuzzifier}

\subsection{General Solitary Collapsed Slice Lemma}

In this paper, we shall derive the RES for the generalised type-2 fuzzy set, $\tilde{F}$. We may think of $\tilde{F}$ as having been formed by repeatedly blurring the membership function of a type-1 fuzzy set $(A)$ at a single domain value $x_{I}$. This gives $n(n \geq 2)$ primary grades $B_{0}^{I}\left(=\mu_{A}\left(x_{I}\right)\right), B_{1}^{I}, B_{2}^{I}, \ldots, B_{n-1}^{I}$ at distances $b_{0}^{I}(=0), b_{1}^{I}, b_{2}^{I}, \ldots, b_{n-1}^{I}$ from $\mu_{A}\left(x_{I}\right)$, to which secondary membership grades $z_{0}^{I}, z_{1}^{I}, z_{2}^{I}, \ldots, z_{n-1}^{I}$ 
are assigned, so turning the Primary Membership Grades (PMGs) into type-2 embedded sets $\tilde{B}_{0}^{I}(=$ $\left.\mu_{A}\left(x_{I}\right)\right), \tilde{B}_{1}^{I}, \tilde{B}_{2}^{I}, \ldots, \tilde{B}_{n-1}^{I}$. Note that $A\left(=B_{0}^{I}\right)$ itself is assigned a secondary membership grade $z_{0}^{I}$, so becoming type- 2 embedded set $\tilde{A}\left(=\tilde{B}_{0}^{I}\right)$. This is illustrated in Fig. 4.

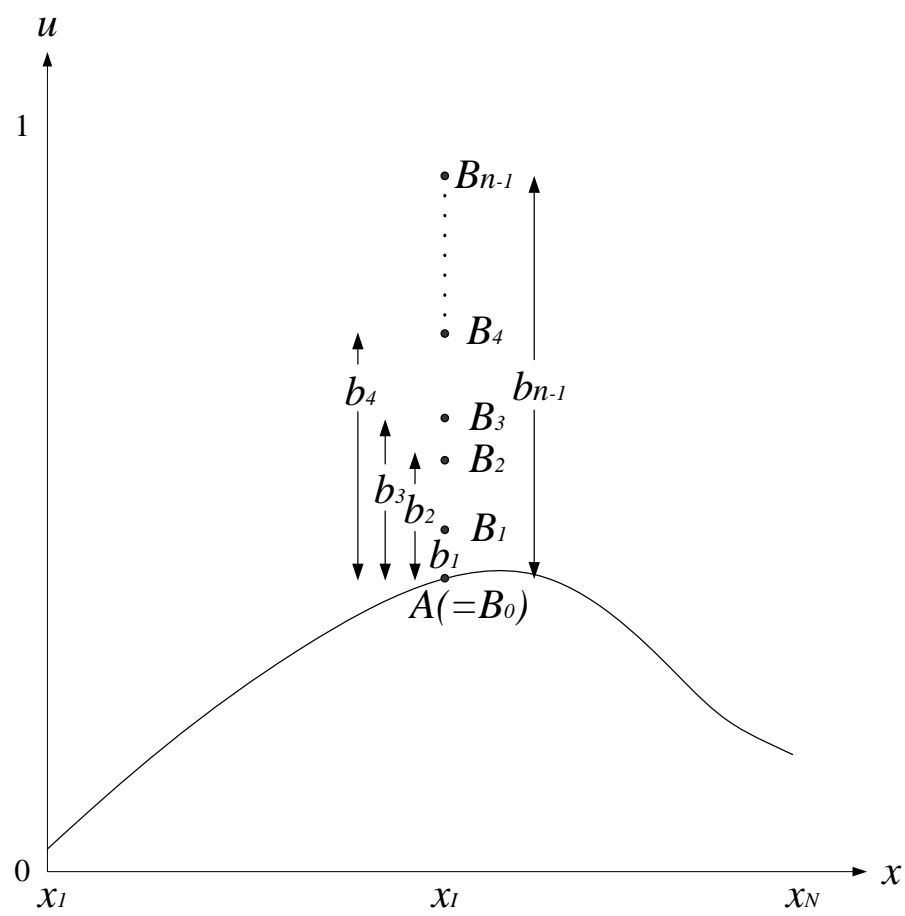

Figure 4: A vertical slice, discretised into more than two co-domain points.

Our objective is to derive a formula for the membership function corresponding to the RES of this generalised type- 2 fuzzy set $\tilde{F}$, in terms of the original type- 1 membership function of the type- 2 embedded set $\tilde{A}, b_{1}^{I}, b_{2}^{I}, \ldots, b_{n-1}^{I}$, and $z_{0}^{I}, z_{1}^{I}, z_{2}^{I}, \ldots, z_{n-1}^{I}$.

$\tilde{F}$ contains $n$ embedded sets, namely $\tilde{A}\left(=\tilde{B}_{0}^{I}\right), \tilde{B}_{1}^{I}, \tilde{B}_{2}^{I}, \ldots, \tilde{B}_{n-1}^{I}$. In the interval case, since all the secondary membership grades are 1 , we are able to find the defuzzified value of $\tilde{F}$ by calculating the mean of $X_{A}$ and $X_{B_{1}}{ }^{I}, X_{B_{2}}{ }^{I}, \ldots, X_{B_{n-1}^{I}}$, (where $X_{B_{i}{ }^{I}}$ is the defuzzified value of $\tilde{B}_{i}^{I}$ ). The generalised type-2 set is more complex; in this case we have to defuzzify the vertical slice by finding its centroid.

Lemma 1 (General Solitary Collapsed Slice Lemma). Let $\tilde{F}$ be the type-2 fuzzy set formed by (upwardly) blurring the membership function of a type-1 fuzzy set (A) at a single domain value $x_{I}$, to create a vertical slice which is an interval as opposed to a single point $\left(\mu_{A}\left(x_{I}\right)\right)$, discretised with $n(n \geq 2)$ points $\left\{B_{0}^{I}, B_{1}^{I}, B_{2}^{I}, \ldots, B_{n-1}^{I}\right\}$ with primary membership grades at distances $b_{0}^{I}(=0), b_{1}^{I}, b_{2}^{I}, \ldots, b_{n-1}^{I}$ from $\mu_{A}\left(x_{I}\right)$, associated with secondary membership grades $\left\{z_{0}^{I}, z_{1}^{I}, z_{2}^{I}, \ldots, z_{n-1}^{I}\right\}$. Let the secondary membership grades of the domain values of the type-1 fuzzy set $(A)\left\{x_{1}, x_{2}, \ldots, x_{I-1}, x_{I}, x_{I+1}, \ldots, x_{N}\right\}$ be denoted as $\left\{z_{0}^{1}, z_{0}^{2}, \ldots, z_{0}^{I-1}, z_{0}^{I}, z_{0}^{I+1}, \ldots, z_{0}^{N}\right\}$. Then $R$, the RES of $\tilde{F}$, has primary membership function

$$
\mu_{R}\left(x_{j}\right)= \begin{cases}\mu_{A}\left(x_{I}\right)+r_{I} & \text { if } j=I, \\ \mu_{A}\left(x_{j}\right) & \text { otherwise, }\end{cases}
$$


where $r_{I}=\frac{\|A\| \sum_{i=0}^{n-1} \frac{b_{i}^{I} Z_{\tilde{B}_{i}^{I}}}{\|A\|+b_{i}^{I}}}{\left\|\tilde{B}_{I}\right\|-\sum_{i=0}^{n-1} \frac{b_{i}^{I} Z_{\tilde{B}_{i}^{I}}}{\|A\|+b_{i}^{I}}} ; Z_{\tilde{B}_{i}^{I}}=\min \left\{z_{0}^{1}, z_{0}^{2}, \ldots, z_{0}^{I-1}, z_{i}^{I}, z_{0}^{I+1}, z_{0}^{I+2}, \ldots, z_{0}^{N}\right\} ;\left\|\tilde{B}_{I}\right\|=\sum_{i=0}^{n-1} Z_{\tilde{B}_{i}^{I}} ;$ and

the following secondary membership grades $\left\{z_{R}^{1}, z_{R}^{2}, \ldots, z_{R}^{I-1}, z_{R}^{I}, z_{R}^{I+1}, \ldots, z_{R}^{N}\right\}$

$$
z_{R}^{j}= \begin{cases}\frac{\left\|\tilde{B}_{I}\right\|}{{ }^{n}} & \text { if } j=I, \\ z_{0}^{j} & \text { otherwise, }\end{cases}
$$

Proof. Let type- 2 fuzzy set $\tilde{R}$ be the RES of $\tilde{F}$ such that its primary membership function $R$ is the same as that of $A$ for all domain values $x_{i}$ apart from $x_{I}{ }^{5}$. At $x_{I}$ the primary membership grade deviates from that of $A$ so that $\mu_{R}\left(x_{I}\right)$ takes the value $\mu_{A}\left(x_{I}\right)+r_{I}$. We know (from [20]) that

$$
X_{R}=X_{A}+\frac{r_{I}\left(x_{I}-X_{A}\right)}{\|A\|+r_{I}} .
$$

We need another expression for $X_{R}$. By definition, $X_{R}=X_{\tilde{F}}$. So we shall proceed by defuzzifying $\tilde{F}$ to find $X_{\tilde{F}}$.

$$
\begin{aligned}
X_{\tilde{F}}= & \text { centroid of TRS of } \tilde{F} \\
= & \text { centroid of }\left\{\left(X_{B_{0}^{I}}, Z_{\tilde{B}_{0}^{I}}\right),\left(X_{B_{1}^{I}}, Z_{\tilde{B}_{1}^{I}}\right),\left(X_{B_{2}^{I}}, Z_{\tilde{B}_{2}^{I}}\right), \ldots,\left(X_{B_{n-1}^{I}}, Z_{\tilde{B}_{n-1}^{I}}\right)\right\} \\
= & \frac{\sum_{i=0}^{n-1} X_{B_{i}^{I}} Z_{\tilde{B}_{i}^{I}}}{\sum_{i=0}^{n-1} Z_{\tilde{B}_{i}^{I}}}
\end{aligned}
$$

where $Z_{\tilde{B}_{i}^{I}}$ is the minimum secondary grade of type- 2 embedded set $B_{i}^{I}$, and may be calculated thus:

$$
Z_{\tilde{B}_{i}^{I}}=\min \left\{z_{0}^{1}, z_{0}^{2}, \ldots, z_{0}^{I-1}, z_{i}^{I}, z_{0}^{I+1}, z_{0}^{I+2}, \ldots, z_{0}^{N}\right\}
$$

where $N$ is the number of vertical slices.

Substituting

$$
X_{B_{i}^{I}}=X_{A}+\frac{b_{i}^{I}\left(x_{I}-X_{A}\right)}{\|A\|+b_{i}^{I}}
$$

we obtain

$$
X_{\tilde{F}}=\frac{\sum_{i=0}^{n-1}\left(X_{A}+\frac{b_{i}^{I}\left(x_{I}-X_{A}\right)}{\|A\|+b_{i}^{I}}\right) Z_{\tilde{B}_{i}^{I}}}{\sum_{i=0}^{n-1} Z_{\tilde{B}_{i}^{I}}}
$$

\footnotetext{
${ }^{5}$ The RES set $\tilde{R}$ of $\tilde{F}$ is a type-2 fuzzy set with singleton primary membership values with an associated secondary membership grade. Thus the reduction step here applies to the fuzzy primary grades being replaced by a representative singleton grade that will provide a centroid for $\tilde{R}$ equal to the centroid of $\tilde{F}$.
} 
Let $\left\|\tilde{B}_{I}\right\|=\sum_{i=0}^{n-1} Z_{\tilde{B}_{i}^{I}}$. Then

$$
X_{\tilde{F}}=\frac{\sum_{i=0}^{n-1}\left(X_{A}+\frac{b_{i}^{I}\left(x_{I}-X_{A}\right)}{\|A\|+b_{i}^{I}}\right) Z_{\tilde{B}_{i}^{I}}}{\left\|\tilde{B}_{I}\right\|} .
$$

Equating $X_{R}$ to $X_{\tilde{F}}$ allows us to obtain a formula for $r_{I}$ :

$$
\begin{aligned}
X_{R}=X_{\tilde{F}} & \Rightarrow X_{A}+\frac{r_{I}\left(x_{I}-X_{A}\right)}{\|A\|+r_{I}}=\frac{1}{\left\|\tilde{B}_{I}\right\|} \sum_{i=0}^{n-1}\left(X_{A}+\frac{b_{i}^{I}\left(x_{I}-X_{A}\right)}{\|A\|+b_{i}^{I}}\right) Z_{\tilde{B}_{i}^{I}} \\
& \Rightarrow X_{A}\left\|\tilde{B}_{I}\right\|+\frac{r_{I}\left(x_{I}-X_{A}\right)\left\|\tilde{B}_{I}\right\|}{\|A\|+r_{I}}=\sum_{i=0}^{n-1}\left(X_{A} Z_{\tilde{B}_{i}^{I}}+\frac{b_{i}^{I}\left(x_{I}-X_{A}\right) Z_{\tilde{B}_{i}^{I}}}{\|A\|+b_{i}^{I}}\right) \\
& \Rightarrow X_{A}\left\|\tilde{B}_{I}\right\|+\frac{r_{I}\left(x_{I}-X_{A}\right)\left\|\tilde{B}_{I}\right\|}{\|A\|+r_{I}}=X_{A}\left\|\tilde{B}_{I}\right\|+\sum_{i=0}^{n-1}\left(\frac{b_{i}^{I}\left(x_{I}-X_{A}\right) Z_{\tilde{B}_{i}^{I}}}{\|A\|+b_{i}^{I}}\right) \\
& \Rightarrow \frac{r_{I}}{\|A\|+r_{I}}=\frac{1}{\left\|\tilde{B}_{I}\right\|} \sum_{i=0}^{n-1} \frac{b_{i}^{I} Z_{\tilde{B}_{i}^{I}}}{\|A\|+b_{i}^{I}} \\
& \Rightarrow\left\|\tilde{B}_{I}\right\| r_{I}=\|A\| \sum_{i=0}^{n-1} \frac{b_{i}^{I} Z_{\tilde{B}_{i}^{I}}}{\|A\|+b_{i}^{I}}+r_{I} \sum_{i=0}^{n-1} \frac{b_{i}^{I} Z_{\tilde{B}_{i}^{I}}}{\|A\|+b_{i}^{I}} \\
& \Rightarrow r_{I}\left(\left\|\tilde{B}_{I}\right\|-\sum_{i=0}^{n-1} \frac{b_{i}^{I} Z_{\tilde{B}_{i}^{I}}}{\|A\|+b_{i}^{I}}\right)=\|A\| \sum_{i=0}^{n-1} \frac{b_{i}^{I} Z_{\tilde{B}_{i}^{I}}}{\|A\|+b_{i}^{I}} \\
& \Rightarrow r_{I}=\frac{\|A\| \sum_{i=0}^{n-1} \frac{b_{i}^{I} Z_{\tilde{B}_{i}^{I}}}{\|A\|+b_{i}^{I}}}{\left\|\tilde{B}_{I}\right\|-\sum_{i=0}^{n-1} \frac{b_{i}^{I} Z_{\tilde{B}_{i}^{I}}}{\|A\|+b_{i}^{I}}}
\end{aligned}
$$

Notice that we also need to find the expression of the secondary membership grade corresponding to the primary membership grade for domain value $x_{I}$ of $\tilde{R}$, the type-2 fuzzy RES set of $\tilde{F}$. Applying the concept of centroid to the second coordinate of the set of points

$$
\left\{\left(X_{B_{0}^{I}}, Z_{\tilde{B}_{0}^{I}}\right),\left(X_{B_{1}^{I}}, Z_{\tilde{B}_{1}^{I}}\right),\left(X_{B_{2}^{I}}, Z_{\tilde{B}_{2}^{I}}\right), \ldots,\left(X_{B_{n-1}^{I}}, Z_{\tilde{B}_{n-1}^{I}}\right)\right\}
$$

we obtain:

$$
z_{R}^{I}=\frac{\sum_{i=0}^{n-1} Z_{\tilde{B}_{i}^{I}}}{n}=\frac{\left\|\tilde{B}_{I}\right\|}{n} .
$$

This completes the proof of the General Solitary Collapsed Slice Lemma (GSCSL).

\subsection{General Representative Embedded Set Approximation}

Corresponding to the GSCSL, the General Representative Embedded Set Approximation (GRESA) is obtained following a similar line of reasoning to that employed in Sections 3 and 4 of [20]. Indeed, a recursive procedure based on the application of Lemma 1 can be used to approximate the representative embedded set $\tilde{R}$ of a general type- 2 fuzzy set. 
Theorem 2 (General Representative Embedded Set Approximation). Let $\tilde{F}$ be a generalised type-2 fuzzy set with lower and upper membership functions $\mu_{L}$ and $\mu_{U}$, whose domain $X$ is discretised into $N$ vertical slices at domain values $\left\{x_{1}, x_{2}, \ldots, x_{I-1}, x_{I}, x_{I+1}, \ldots, x_{N}\right\}$ with secondary membership grades (corresponding to the lower membership function $\mu_{L}$ ) be denoted as $\left\{z_{0}^{1}, z_{0}^{2}, \ldots, z_{0}^{I-1}, z_{0}^{I}, z_{0}^{I+1}, \ldots, z_{0}^{N}\right\}$. Assume that each vertical slice is discretised between $L$ and $U$ using $n(n \geq 2)$ points $\left\{B_{0}^{I}, B_{1}^{I}, B_{2}^{I}, \ldots, B_{n-1}^{I}\right\}$, with primary membership grades at distances $b_{0}^{I}(=0), b_{1}^{I}, b_{2}^{I}, \ldots, b_{n-1}^{I}\left(=\mu_{U}\left(x_{I}\right)-\mu_{L}\left(x_{I}\right)\right)$ from $\mu_{L}\left(x_{I}\right)$ to which secondary membership grades $\left\{z_{0}^{I}, z_{1}^{I}, z_{2}^{I}, \ldots, z_{n-1}^{I}\right\}$ are assigned. The primary membership function of the general representative embedded set approximation $\tilde{R}$ is:

$$
\begin{array}{ll}
\forall I=1, \ldots, N: \quad \mu_{\tilde{R}}\left(x_{I}\right)=\mu_{L}\left(x_{I}\right)+r_{I} ; \\
r_{I}=\frac{\left\|R_{I-1}\right\| \sum_{i=0}^{n-1} \frac{b_{i}^{I} Z_{\tilde{B}_{i}^{I}}}{\left\|R_{I-1}\right\|+b_{i}^{I}}}{\left\|\tilde{B}_{I}\right\|-\sum_{i=0}^{n-1} \frac{b_{i}^{I} Z_{\tilde{B}_{i}^{I}}}{\left\|R_{I-1}\right\|+b_{i}^{I}}} \\
\left\|R_{I-1}\right\|=\|L\|+\sum_{k=1}^{I-1} r_{k} ;\left(\left\|R_{0}\right\|=\|L\|\right) ; \\
\left\|\tilde{B}_{I}\right\|=\sum_{i=0}^{n-1} Z_{\tilde{B}_{i}^{I}} ; \\
Z_{\tilde{B}_{i}^{I}}=\min \left\{z_{\tilde{R}}^{1}, z_{\tilde{R}}^{2}, \ldots, z_{\tilde{R}}^{I-1}, z_{i}^{I}, z_{0}^{I+1}, z_{0}^{I+2}, \ldots, z_{0}^{N}\right\} ;
\end{array}
$$

and corresponding secondary membership grade

$$
z_{\tilde{R}}^{I}=\frac{\left\|\tilde{B}_{I}\right\|}{n}
$$

Proof. In its first step, Lemma 1 is applied to compute the RES, $R_{1}$, of the type- 2 fuzzy set formed by (upwardly) blurring the lower membership functions $\mu_{L}$ at $x_{1}$ to create vertical slice 1 , discretised with $n(n \geq 2)$ points $\left\{B_{0}^{1}, B_{1}^{1}, B_{2}^{1}, \ldots, B_{n-1}^{1}\right\}$ with primary membership grades at distances $b_{0}^{1}(=0), b_{1}^{1}, b_{2}^{1}, \ldots$, $b_{n-1}^{1}\left(=\mu_{U}\left(x_{1}\right)-\mu_{L}\left(x_{1}\right)\right)$ from $\mu_{A}\left(x_{1}\right)$, associated with secondary membership grades $\left\{z_{0}^{1}, z_{1}^{1}, z_{2}^{1}, \ldots, z_{n-1}^{1}\right\}$. In this step, we use secondary membership grades $\left\{z_{0}^{1}, z_{0}^{2}, z_{0}^{3}, \ldots, z_{0}^{N}\right\}$ to compute $R_{1}$. In the second step, we apply Lemma 1 to the type-2 fuzzy set formed by (upwardly) blurring the lower membership functions $\mu_{R-1}$ at $x_{2}$, and compute its RES, $R_{1}$, etc..

In what follows, we will use induction on the number of collapsing vertical slices in conjunction with Lemma 1 to prove the theorem.

1. Basis (collapsing vertical slice at $x_{1}$ ): We apply Lemma 1 to the type- 2 fuzzy set formed by (upwardly) blurring the lower membership functions $\mu_{L}$, with secondary membership grades $\left\{z_{0}^{1}, z_{0}^{2}, z_{0}^{3}, \ldots, z_{0}^{N}\right\}$, at $x_{1}$ to create vertical slice 1 , discretised with points $\left\{B_{0}^{1}, B_{1}^{1}, B_{2}^{1}, \ldots, B_{n-1}^{1}\right\}$ with primary membership grades at distances $b_{0}^{1}(=0), b_{1}^{1}, b_{2}^{1}, \ldots, b_{n-1}^{1}\left(=\mu_{U}\left(x_{1}\right)-\mu_{L}\left(x_{1}\right)\right)$ from $\mu_{A}\left(x_{1}\right)$, associated with secondary membership grades $\left\{z_{0}^{1}, z_{1}^{1}, z_{2}^{1}, \ldots, z_{n-1}^{1}\right\}$. The RES, $R_{1}$, of this type-2 fuzzy set has the following primary membership function

$$
\mu_{R_{1}}\left(x_{j}\right)= \begin{cases}\mu_{L}\left(x_{1}\right)+r_{1} & \text { if } j=1 \\ \mu_{L}\left(x_{j}\right) & \text { otherwise }\end{cases}
$$


where $r_{1}=\frac{\|L\| \sum_{i=0}^{n-1} \frac{b_{i}^{1} Z_{\tilde{B}_{i}^{1}}}{\|L\|+b_{i}^{1}}}{\left\|\tilde{B}_{1}\right\|-\sum_{i=0}^{n-1} \frac{b_{i}^{1} Z_{\tilde{B}_{i}^{1}}}{\|L\|+b_{i}^{1}}} ; Z_{\tilde{B}_{i}^{1}}=\min \left\{z_{i}^{1}, z_{0}^{2}, \ldots, z_{0}^{N}\right\} ;\left\|\tilde{B}_{I}\right\|=\sum_{i=0}^{n-1} Z_{\tilde{B}_{i}^{1}} ;$ and the following

secondary membership grades

$$
z_{R_{1}}^{j}= \begin{cases}\frac{\left\|\tilde{B}_{1}\right\|}{{ }^{j}} & \text { if } j=1 \\ z_{0} & \text { otherwise. }\end{cases}
$$

Because $\left\|R_{0}\right\|=\|L\|$ and $z_{R_{1}}^{1}=z_{\tilde{R}}^{1}, \mu_{R_{1}}\left(x_{1}\right)=\mu_{\tilde{R}}\left(x_{1}\right)$.

2. Induction hypothesis: Assume that the type-2 fuzzy set formed by (upwardly) blurring the lower membership functions $\mu_{R_{I-1}}$, with corresponding secondary membership grades $\left\{z_{\tilde{R}}^{1}, z_{\tilde{R}}^{2}, \ldots, z_{\tilde{R}}^{I-1}, z_{R_{I-1}}^{I}(=\right.$ $\left.\left.z_{0}^{I}\right), z_{R_{I-1}}^{I+1}\left(=z_{0}^{I+1}\right), \ldots, z_{R_{I-1}}^{N}\left(=z_{0}^{N}\right)\right\}$, at $x_{I}$ to create vertical slice I, which is discretised with the $n$ points $\left\{B_{0}^{I}, B_{1}^{I}, B_{2}^{I}, \ldots, B_{n-1}^{I}\right\}$ with primary membership grades at distances $b_{0}^{I}(=0), b_{1}^{I}, b_{2}^{I}, \ldots$, $b_{n-1}^{I}\left(=\mu_{U}\left(x_{I}\right)-\mu_{L}\left(x_{I}\right)\right)$ from $\mu_{A}\left(x_{I}\right)$, associated with following secondary membership grades $\left\{z_{0}^{I}, z_{1}^{I}, z_{2}^{I}, \ldots, z_{n-1}^{I}\right\}$. Then, applying Lemma 1 , the RES, $R_{I}$, of this type-2 fuzzy set has following primary membership function

$$
\mu_{R_{I}}\left(x_{j}\right)= \begin{cases}\mu_{R_{I-1}}\left(x_{I}\right)+r_{I} & \text { if } j=I \\ \mu_{R_{I-1}}\left(x_{j}\right) & \text { otherwise }\end{cases}
$$

where $r_{I}=\frac{\left\|R_{I-1}\right\| \sum_{i=0}^{n-1} \frac{b_{i}^{I} Z_{\tilde{B}_{i}^{I}}}{\left\|R_{I-1}\right\|+b_{i}^{I}}}{\left\|\tilde{B}_{I}\right\|-\sum_{i=0}^{n-1} \frac{b_{i}^{I} Z_{\tilde{B}_{i}^{I}}}{\left\|R_{I-1}\right\|+b_{i}^{I}}} ; Z_{\tilde{B}_{i}^{I-1}}=\min \left\{z_{\tilde{R}}^{1}, z_{\tilde{R}}^{2}, \ldots, z_{\tilde{R}}^{I-1}, z_{i}^{I}, z_{0}^{I+1} \ldots, z_{0}^{N}\right\} ;\left\|\tilde{B}_{I}\right\|=\sum_{i=0}^{n-1} Z_{\tilde{B}_{i}^{I}} ;$

and the following secondary membership grades

$$
z_{R_{I}}^{j}= \begin{cases}\frac{\left\|\tilde{B}_{I}\right\|}{n} & \text { if } j=I \\ z_{R_{I-1}^{j}}^{n} & \text { otherwise. }\end{cases}
$$

It is clear that $\mu_{R_{I}}\left(x_{I}\right)=\mu_{\tilde{R}}\left(x_{I}\right)$.

3. Conclusion: The described recursive procedure based on the application of Lemma 1 proves that the given primary membership function of the general representative embedded set approximation $\tilde{R}$ is correct.

After Theorem 2 is applied, we end up with the following type-2 fuzzy set

$$
\tilde{R}=\left\{\left(x_{1}, \mu_{\tilde{R}}\left(x_{1}\right), z_{\tilde{R}}^{1}\right),\left(x_{2}, \mu_{\tilde{R}}\left(x_{2}\right), z_{\tilde{R}}^{2}\right), \ldots,\left(x_{1}, \mu_{\tilde{R}}\left(x_{1}\right), z_{\tilde{R}}^{1}\right)\right\} .
$$

This is called the General Representative Embedded Set Approximation (GRESA) of type-2 fuzzy set $\tilde{F}$. The centroid of $\tilde{R}$ is

$$
X_{\tilde{R}}=\frac{\sum_{j=1}^{N} x_{j} \cdot \mu_{\tilde{R}}\left(x_{j}\right)}{\sum_{j=1}^{N} \mu_{\tilde{R}}\left(x_{j}\right)} .
$$




\subsubsection{The GRESA Algorithm}

Theorem 2 may be outlined algorithmically:

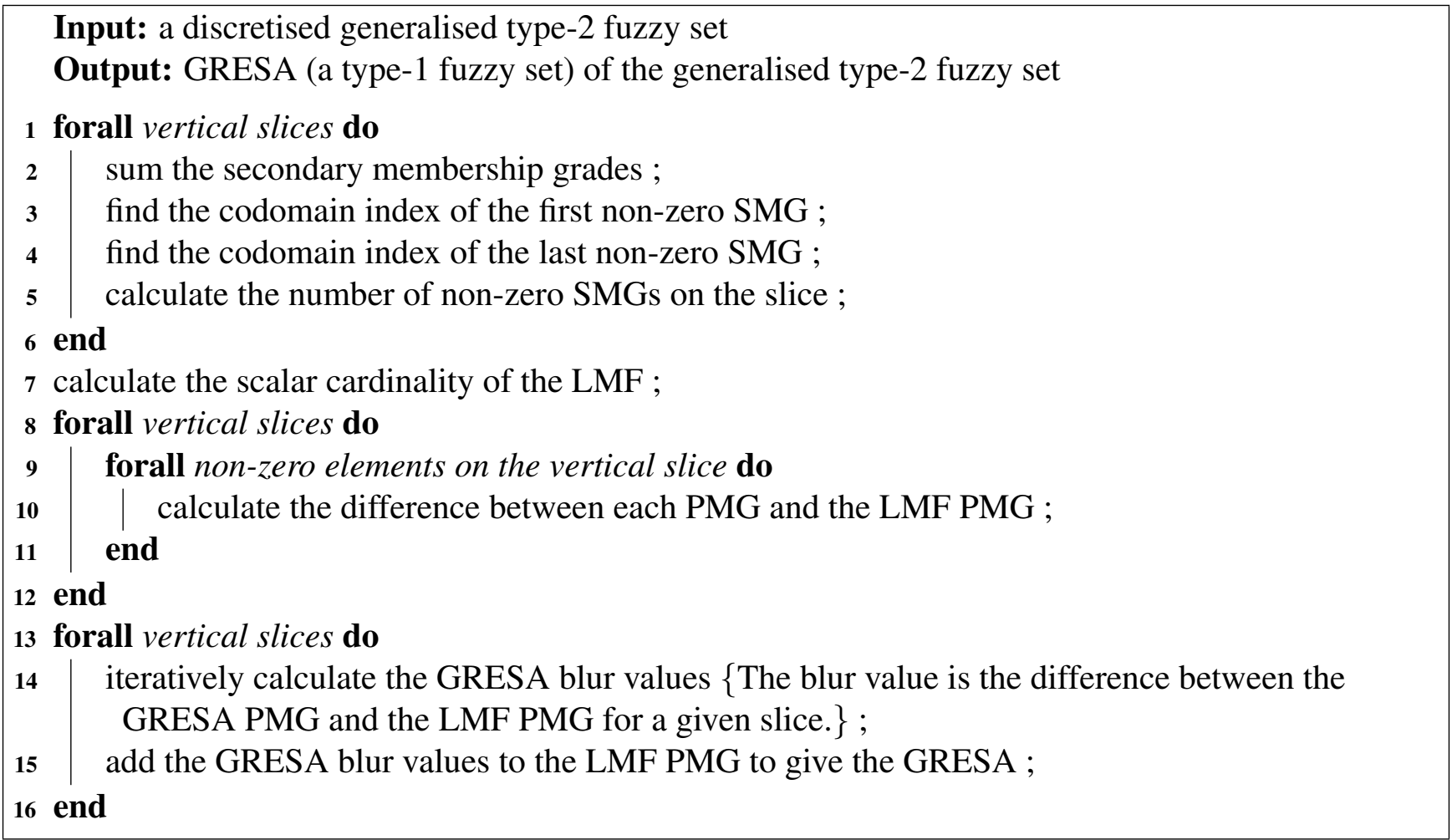

\section{Algorithm 2: GRESA}

\section{Testing the GGCCD}

This section reports on tests contrasting the GGCCD and $\alpha$-Planes/KMIP Method (Subsection 2.3.3) for accuracy and efficiency relative to the absolutely accurate but inefficient Exhaustive Method of Defuzzification (Subsection 2.1). The error is defined as the difference between the Exhaustive Method defuzzified value and the defuzzified value obtained by the method being tested. As regards efficiency, the speed improvement factor is defined as the factor by which the speed of processing is multiplied by adopting the method under test. It is calculated by dividing the Exhaustive Defuzzification time by the defuzzification time of the method under test.

\subsection{Experimental Set-Up}

The defuzzification methods were coded in Matlab ${ }^{T M} \mathrm{R} 2014 \mathrm{a}$ and tested on a PC with an Intel(R) Core $^{T M}$ i5-4570 CPU and a 8.00 GB RAM, with a clock speed of 3.20 GHz. The operating system used was MS Windows 10 Education. Each test program was run as a process with a priority that was higher than that of the operating system, so as to eliminate, as far as possible, timing errors caused by other operating system processes.

Six generalised type-2 fuzzy test sets were created, depicted in Figures A.5 to A.10. These are aggregated sets output by the inferencing stage of a prototype type- 2 FIS ${ }^{6}$. For each inference the degree

\footnotetext{
${ }^{6}$ The Matlab ${ }^{T M}$ code for test set creation and defuzzification may be found at http://www.tech.dmu.ac.uk/ sarahg/
} 
of discretisation adopted was sufficiently coarse to permit Exhaustive Defuzzification. Three rule sets were used. For each rule set the FIS was run with two distinct sets of parameters ${ }^{7}$. Table 1 summarises the features of the test sets. More information about the rule sets may be found in [15]. The FIS generated test sets were selected because of the complexity and lack of symmetry evident in their graphs; their benchmark defuzzified values were found by Exhaustive Defuzzification. Each test set was defuzzified

- once through Exhaustive Defuzzification;

- 1000 times via the GGCCD;

- 1000 times via the $\alpha$-Planes/KMIP Method using 11 evenly spaced $\alpha$-planes;

- 1000 times via the $\alpha$-Planes/KMIP Method using 101 evenly spaced $\alpha$-planes.

For the tests involving the GGCCD and $\alpha$-Planes/KMIP Method the averages of 1000 timings were taken to allow for the effects of extraneous operating system processes which cannot be totally eliminated. The means and standard deviations of the defuzzification times were calculated ${ }^{8}$.

\begin{tabular}{|l||l|l|l|l|}
\hline $\begin{array}{l}\text { Test } \\
\text { Set }\end{array}$ & Normal & $\begin{array}{l}\text { Normal } \\
\text { Sec. MF }\end{array}$ & $\begin{array}{l}\text { Narrow } \\
\text { FOU }\end{array}$ & $\begin{array}{l}\text { No. of } \\
\text { Emb. Sets }\end{array}$ \\
\hline \hline Heater0.125 & yes & no & no & 14580 \\
Heater0.0625 & yes & no & yes & 13778100 \\
Powder0.1 & yes & no & yes & 24300 \\
Powder0.05 & yes & yes & yes & 3840000 \\
Shopping0.2 & no & no & no & 16 \\
Shopping0.1 & yes & yes & no & 312500 \\
\hline
\end{tabular}

Table 1: Features of the generalised type-2 fuzzy test sets.

\subsection{Results and Discussion}

Table 2 shows the results of the experiments in relation to accuracy, and Table 3 the results in relation to efficiency. The RESA for each test set are shown in Figures A.5 to A.10.

The GGCCD and the $\alpha$-Planes Method are approximate techniques; the experiments show both approaches to produce good approximations, with the $\alpha$-Planes Method the superior of the two as it is more accurate in two thirds of the test cases ${ }^{9}$.

Both the GGCCD and the $\alpha$-Planes/KMIP Method are demonstrated to be very much faster than the Exhaustive Method. However in every instance the GGCCD is shown to be faster than the $\alpha$ Planes/KMIP Method. Where there are $11 \alpha$-planes the GGCCD takes between 55\% and 80\% of the time taken by the $\alpha$-Planes/KMIP Method. The GGCCD is over 10 times faster when the type-2 fuzzy set is decomposed into $101 \alpha$-planes. These efficiencies are of the most consequence in control applications, where rapid execution is of the essence.

\footnotetext{
${ }^{7}$ For example Heater0.0625 is not a finer version of Heater0.125; it uses different parameters for the input rules. That these two test sets differ can be clearly seen from their 3-D representations.

${ }^{8}$ The experiment has two parts: Firstly, the prototype type-2 FIS is applied to create the aggregated type-2 fuzzy output (Figure 1). This aggregated set is then used as a test set. Secondly, the three defuzzification methods to be tested are applied to the aggregated sets. Table 3 provides the timings for this phase of the experiment. Timings solely account for defuzzification; times for creation of the test sets are not taken into account.

${ }^{9}$ Interestingly, these results show the accuracy of the $\alpha$-planes approach is greater when $11 \alpha$-planes are used than when $101 \alpha$-planes are used. This fits in with the observation reported in [15] that as the number of $\alpha$-planes increases, the $\alpha$-Planes Method results converge, but not to the values obtained by Exhaustive Defuzzification.
} 


\begin{tabular}{|l|l|l|l|l|l|l|l|}
\hline Test Set & Exhaustive & GGCCD DV & $\begin{array}{l}\text { GGCCD } \\
\text { EV }\end{array}$ & $\begin{array}{l}11 \alpha \text {-Planes/ } \\
\text { KMIP DV }\end{array}$ & $\begin{array}{l}11 \alpha \text {-Planes/ } \\
\text { KMIP Error }\end{array}$ & $\begin{array}{l}101 \alpha \text {-Planes/ } \\
\text { KMIP DV }\end{array}$ & $\begin{array}{l}101 \alpha \text {-Planes/ } \\
\text { KMIP Error }\end{array}$ \\
\hline \hline Heater0.125 & 0.6313618377 & 0.6459218438 & $\mathbf{0 . 0 1 4 5 6 0 0 0 6 1}$ & 0.6119623264 & 0.0193995113 & 0.6063363068 & 0.0250255309 \\
Heater0.0625 & 0.2621587894 & 0.2267190468 & 0.0354397426 & 0.2734346950 & $\mathbf{0 . 0 1 1 2 7 5 9 0 5 6}$ & 0.2797895424 & 0.0176307530 \\
Powder0.1 & 0.2806983775 & 0.2240503031 & 0.0566480744 & 0.2818122309 & $\mathbf{0 . 0 0 1 1 1 3 8 5 3 4}$ & 0.2868681564 & 0.0061697789 \\
Powder0.05 & 0.8180632180 & 0.8507385436 & 0.0326753256 & 0.8007539910 & $\mathbf{0 . 0 1 7 3 0 9 2 2 7 0}$ & 0.7959566861 & 0.0221065319 \\
Shopping0.2 & 0.5481044441 & 0.5606320492 & $\mathbf{0 . 0 1 2 5 2 7 6 0 5 1}$ & 0.5323554121 & 0.0157490320 & 0.5313901724 & 0.0167142717 \\
Shopping0.1 & 0.5954109472 & 0.6691362079 & 0.0737252607 & 0.5905091249 & $\mathbf{0 . 0 0 4 9 0 1 8 2 2 3}$ & 0.5867273458 & 0.0086836014 \\
\hline \hline
\end{tabular}

Table 2: The defuzzified values from the GGCCD and the $\alpha$-Planes/KMIP Method contrasted with those obtained via the Exhaustive Method. Two variants of the $\alpha$-Planes Decomposition were employed, one with $11 \alpha$-planes and the other with $101 \alpha$-planes. The smaller errors are shown in bold.

\begin{tabular}{|c|c|c|c|c|c|c|c|c|c|c|}
\hline $\begin{array}{l}\text { Test } \\
\text { Set }\end{array}$ & $\begin{array}{l}\text { Exhaustive } \\
\text { Defuzz. } \\
\text { Time }\end{array}$ & $\begin{array}{l}\text { Mean } \\
\text { GGCCD } \\
\text { Defuzz. } \\
\text { Time }\end{array}$ & $\begin{array}{l}\text { SD of } \\
\text { GGCCD } \\
\text { Defuzz. } \\
\text { Time }\end{array}$ & $\begin{array}{l}\text { GGCCD } \\
\text { Speed } \\
\text { Improv. } \\
\text { Factor }\end{array}$ & $\begin{array}{l}\text { Mean } 11 \\
\alpha-P . / K M I P \\
\text { Defuzz. } \\
\text { Time }\end{array}$ & $\begin{array}{l}\text { SD of } 11 \\
\alpha-P . / K M I P \\
\text { Defuzz. } \\
\text { Time }\end{array}$ & $\begin{array}{l}11 \alpha-P . / \\
\text { KMIP Speed } \\
\text { Improv. } \\
\text { Factor }\end{array}$ & $\begin{array}{l}\text { Mean } 101 \\
\alpha-P . / K M I P \\
\text { Defuzz. } \\
\text { Time }\end{array}$ & $\begin{array}{l}\text { SD of } 101 \\
\alpha-P . / K M I P \\
\text { Defuzz. } \\
\text { Time }\end{array}$ & $\begin{array}{l}101 \alpha-P . / \\
\text { KMIP Speed } \\
\text { Improv. } \\
\text { Factor }\end{array}$ \\
\hline Heater0.0625 & 907.073445 & 0.0005052 & 0.0000365 & 1795474.0 & 0.0006456 & 0.0000616 & 1405008.4 & 0.0053977 & 0.0000817 & 168048.1 \\
\hline Powder0.1 & 1.513906 & 0.0003178 & 0.0000310 & 4763.7 & 0.0005914 & 0.0000687 & 2559.9 & 0.0048862 & 0.0001453 & 309.8 \\
\hline Powder0.05 & 278.593608 & 0.0005686 & 0.0000401 & 489964.1 & 0.0007114 & 0.0000611 & 391613.2 & 0.0061578 & 0.0000667 & 45242.4 \\
\hline Shopping0.1 & 20.010074 & 0.0003377 & 0.0000319 & 59254.0 & 0.0006055 & 0.0000482 & 33047.2 & 0.0053419 & 0.0001555 & 3745.9 \\
\hline
\end{tabular}

Table 3: The timings (in seconds) of the GGCCD and the $\alpha$-Planes/KMIP Method contrasted with those of the Exhaustive Method. Two variants of the $\alpha$-Planes Decomposition were employed, one with $11 \alpha$-planes and the other with $101 \alpha$-planes. The greater speed improvement factors are shown in bold. 


\section{Conclusions and Further Work}

This paper contributes to the theory of type-2 fuzzy logic in relation to defuzzification by extending the interval GCCD to generalised type-2 fuzzy sets, resulting in the GGCCD. Experiments reveal good accuracy of the GGCCD with respect to the Exhaustive Method. Moreover it is extremely fast compared with the Exhaustive Method. Regardless of how many $\alpha$-planes are employed, for two thirds of the test cases the GGCCD is less accurate than the $\alpha$-Planes/KMIP Method, but in every case it is faster. In the GGCCD, type-2 fuzzy logic developers now have another defuzzification technique to add to those options presented in Section 2.

Future research will address these issues:

Collapsing Direction For the interval GCCD it was demonstrated that the order of slice collapse has an impact on accuracy (Subsection 3) [16, 21]. There is every reason to suppose that the same improvement of accuracy will occur with the GGCCD, but it would be profitable to investigate further to confirm that this is the case.

Comparison With Existing Methods Greenfield and Chiclana's 2013 paper [15] evaluated and contrasted defuzzification techniques for generalised type-2 fuzzy sets. It is timely to update this research to include the GGCCD among the strategies evaluated. In such a comparison it would be appropriate to improve the timings for the $\alpha$-Planes Method by replacing the original KMIP by a faster enhancement [50].

\section{Appendix A. Test Sets}

\section{References}

[1] J. Aisbett, J. T. Rickard, and D. G. Morgenthaler. Type-2 Fuzzy Sets as Functions on Spaces. IEEE Transactions on Fuzzy Systems, 18(4):841-844, August 2010.

[2] D. Bhattacharya, A. Konar, and P. Das. Secondary factor induced stock index time-series prediction using Self-Adaptive Interval Type-2 Fuzzy Sets. Neurocomputing, 171:551 - 568, 2016.

[3] S. Bhattacharyya, D. Basu, A. Konar, and D. N. Tibarewala. Interval type-2 fuzzy logic based multiclass ANFIS algorithm for real-time EEG based movement control of a robot arm. Robotics and Autonomous Systems, 68:104 - 115, 2015.

[4] S. Bhattacharyya, M. Pal, A. Konar, and D. N. Tibarewala. An interval type-2 fuzzy approach for real-time EEG-based control of wrist and finger movement. Biomedical Signal Processing and Control, 21:90 - 98, 2015.

[5] O. Castillo and P. Melin. A review on interval type-2 fuzzy logic applications in intelligent control. Information Sciences, 279:615 - 631, 2014. doi: http://dx.doi.org/10.1016/j.ins.2014.04.015.

[6] O. Castillo, L. Cervantes, J. Soria, M. Sanchez, and J. R. Castro. A generalized type-2 fuzzy granular approach with applications to aerospace. Information Sciences, 354:165.

[7] J. C. Cortes-Rios, E. Gómez-Ramírez, H. Alejandro Ortiz de-la Vega, O. Castillo, and P. Melin. Optimal design of interval type 2 fuzzy controllers based on a simple tuning algorithm. Applied Soft Computing, 23:270 - 285, 2014. 


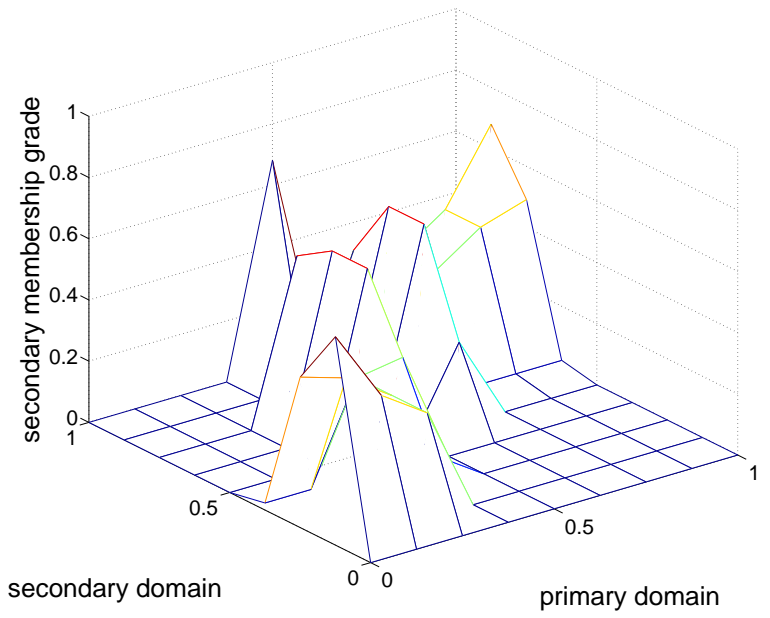

(a) 3-D representation.

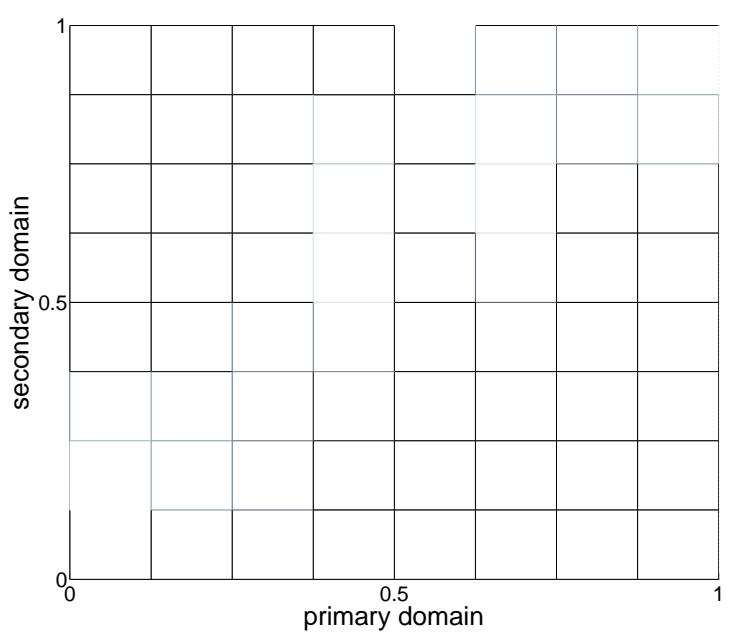

(b) FOU.

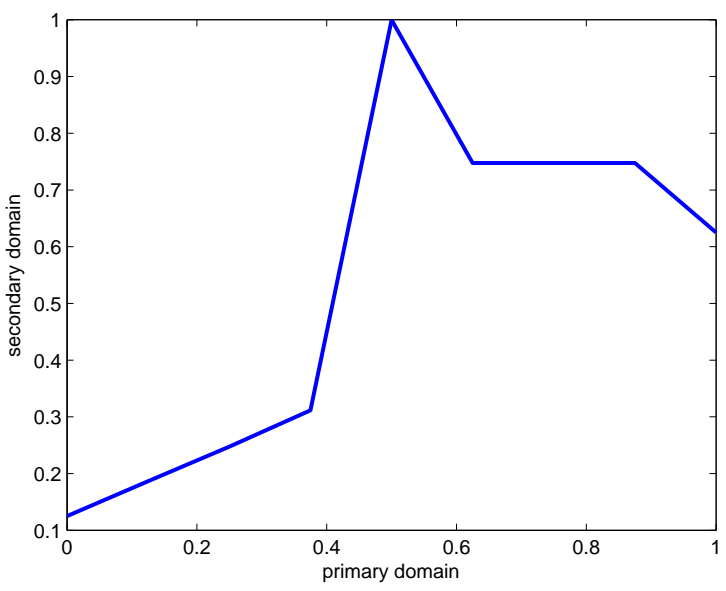

(c) GRESA.

Figure A.5: Heater0.125 - Heater FIS generated generalised test set, domain degree of discretisation 0.125. 


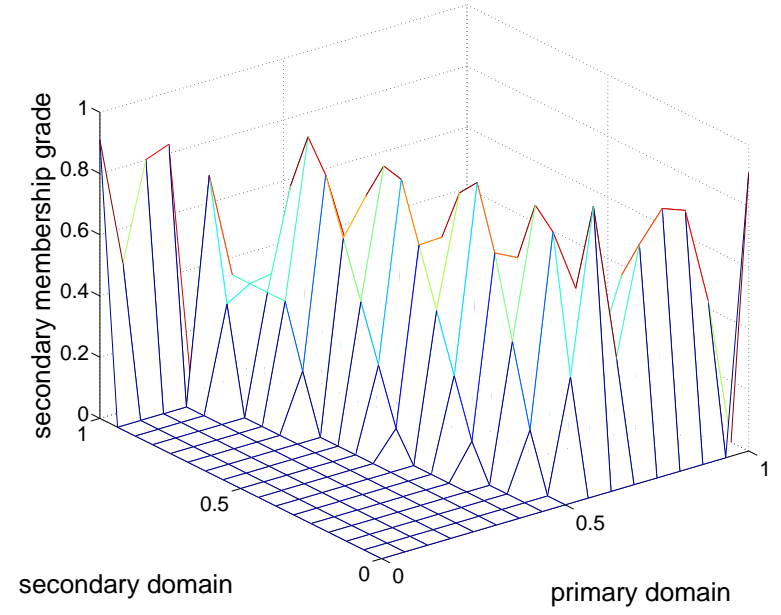

(a) 3-D representation.

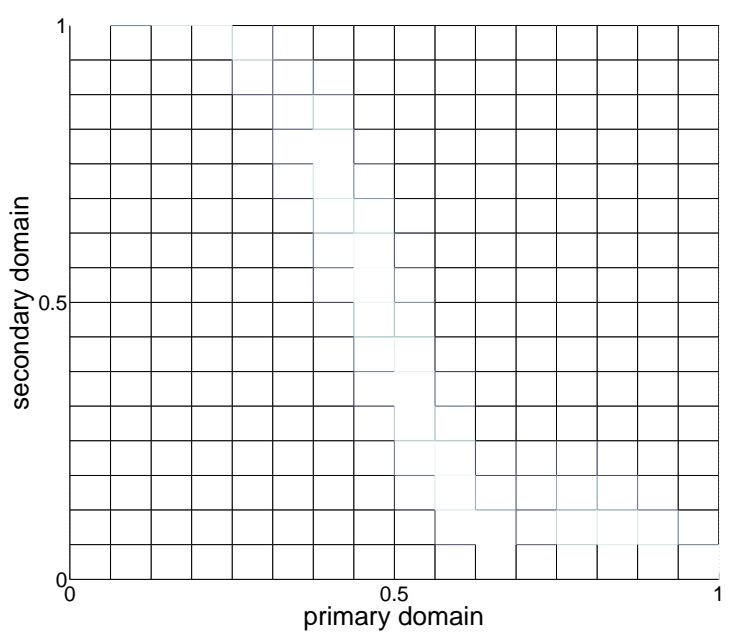

(b) FOU.

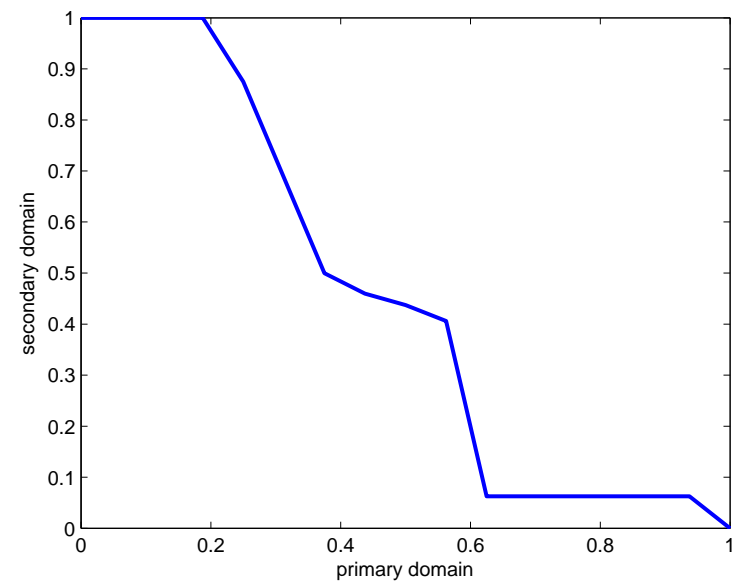

(c) GRESA.

Figure A.6: Heater0.0625 - Heater FIS generated generalised test set, domain degree of discretisation 0.0625. 


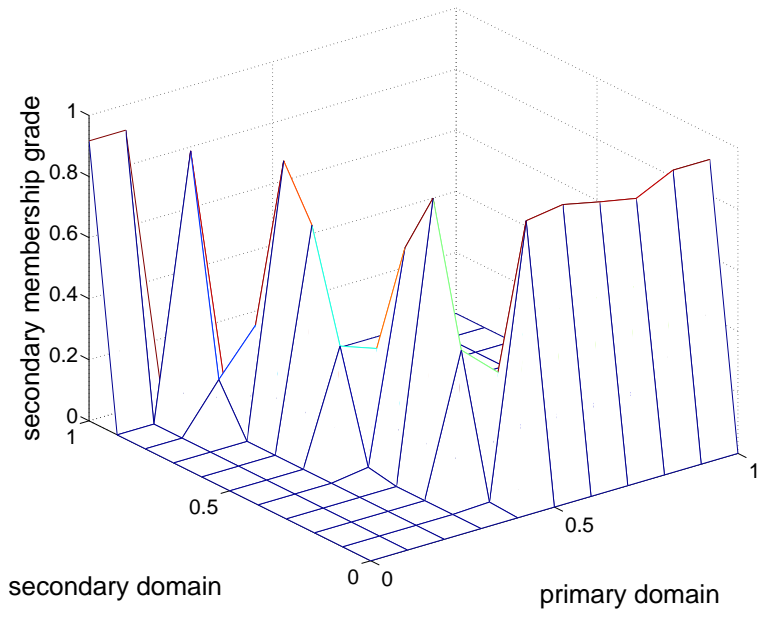

(a) 3-D representation.

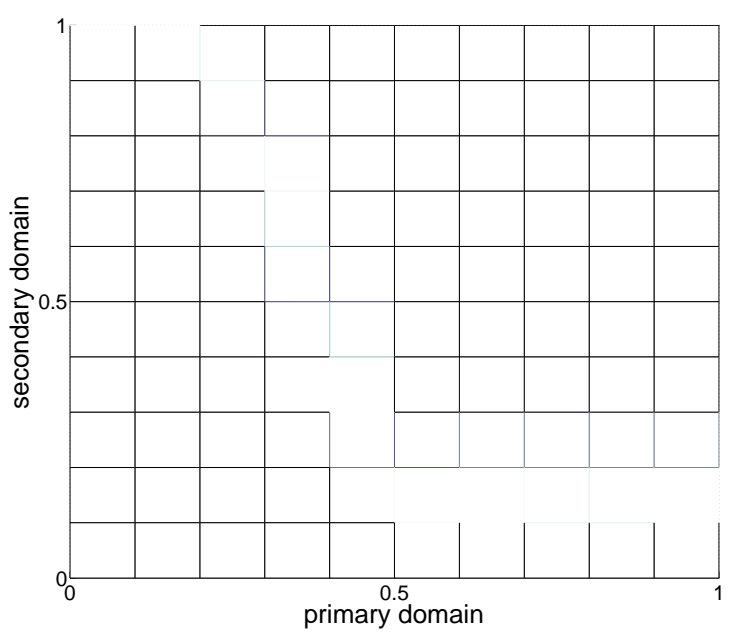

(b) FOU.

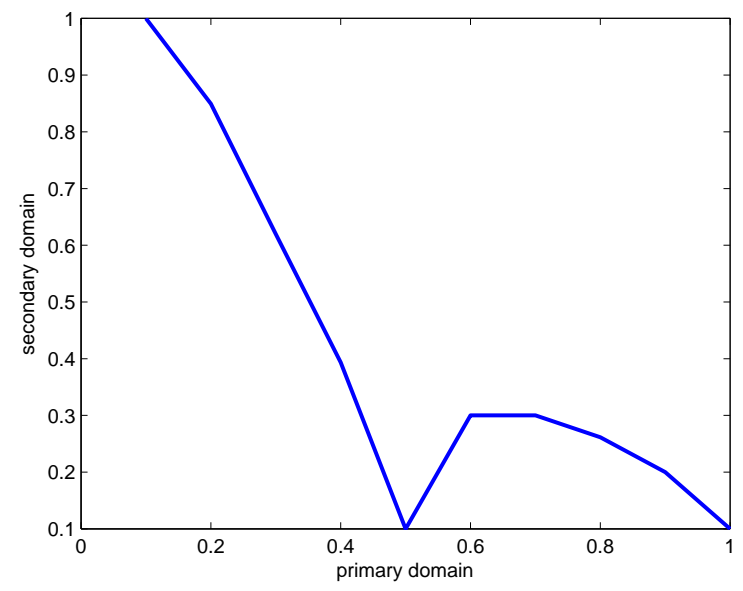

(c) GRESA.

Figure A.7: Powder0.1 — Powder FIS generated generalised test set, domain degree of discretisation 0.1. 


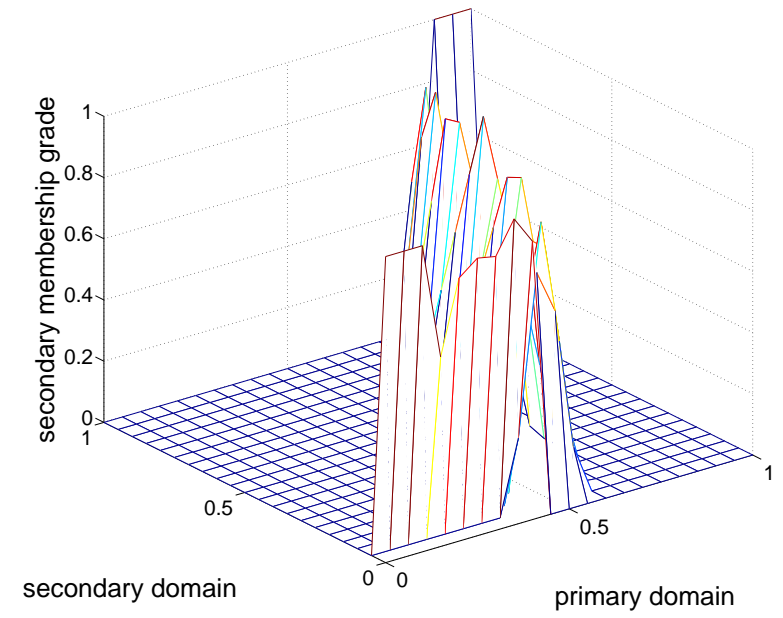

(a) 3-D representation.

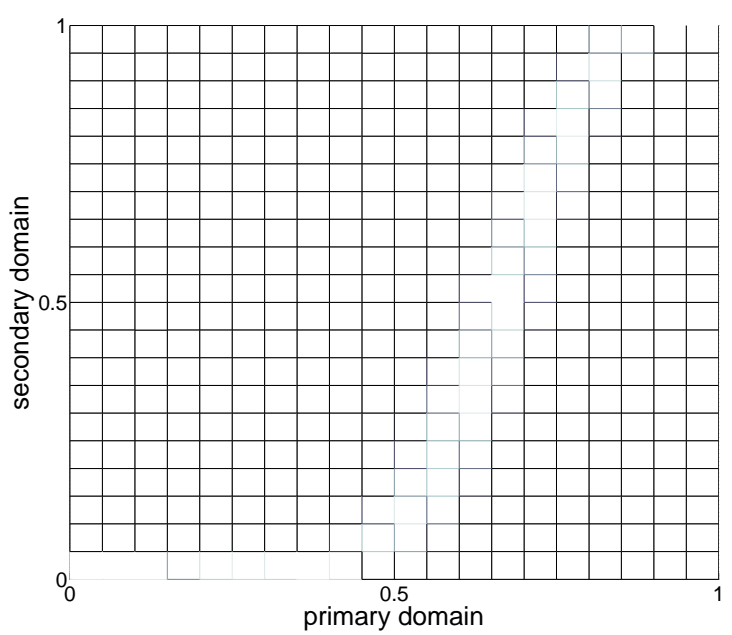

(b) FOU.

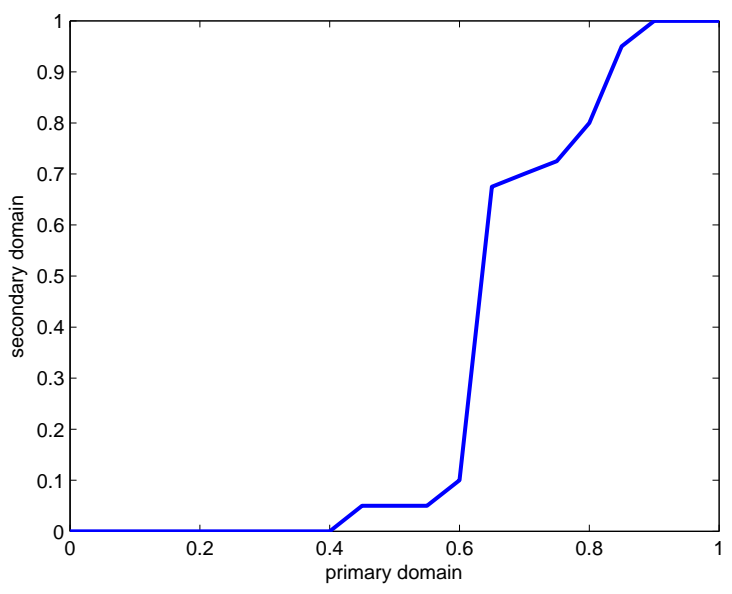

(c) GRESA.

Figure A.8: Powder0.05 - Powder FIS generated generalised test set, domain degree of discretisation 0.05. 


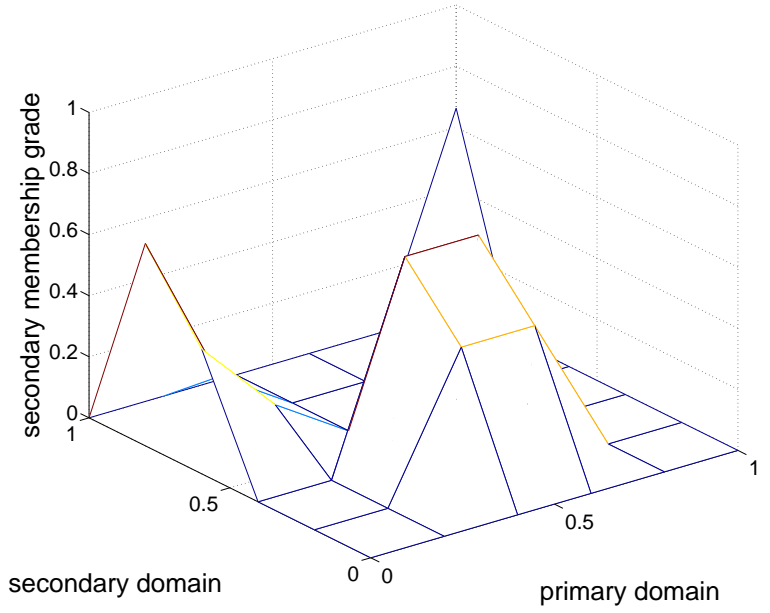

(a) 3-D representation.

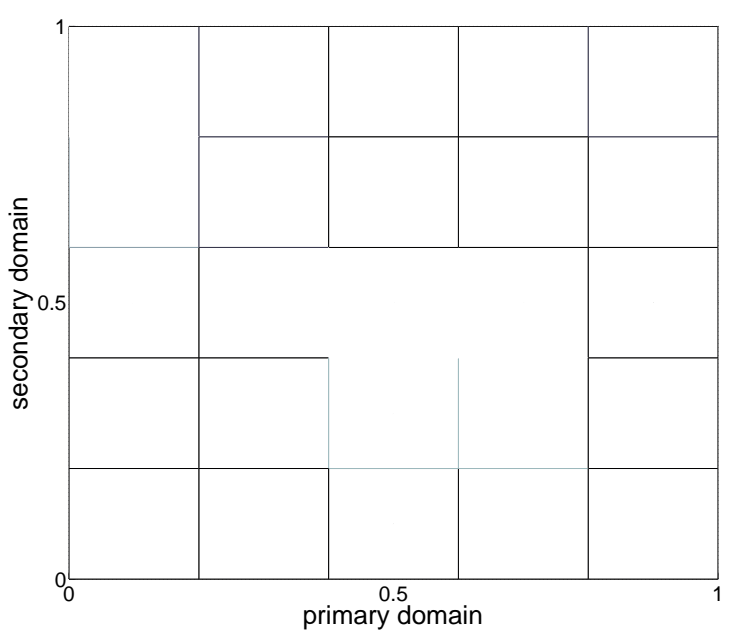

(b) FOU.

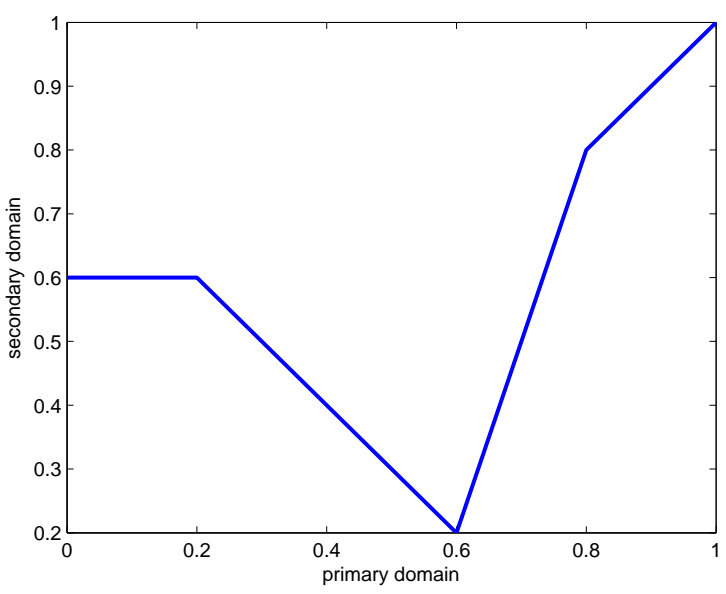

(c) GRESA.

Figure A.9: Shopping0.2 — Shopping FIS generated generalised test set, domain degree of discretisation 0.2. 


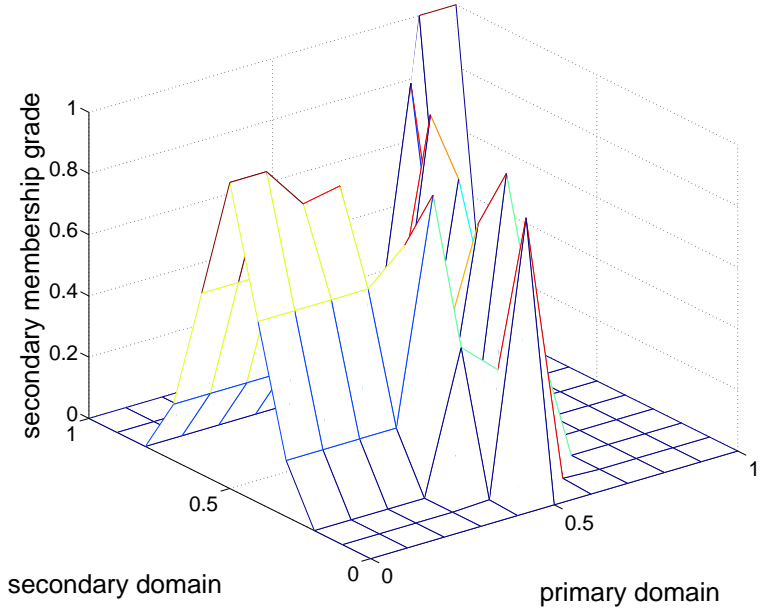

(a) 3-D representation.

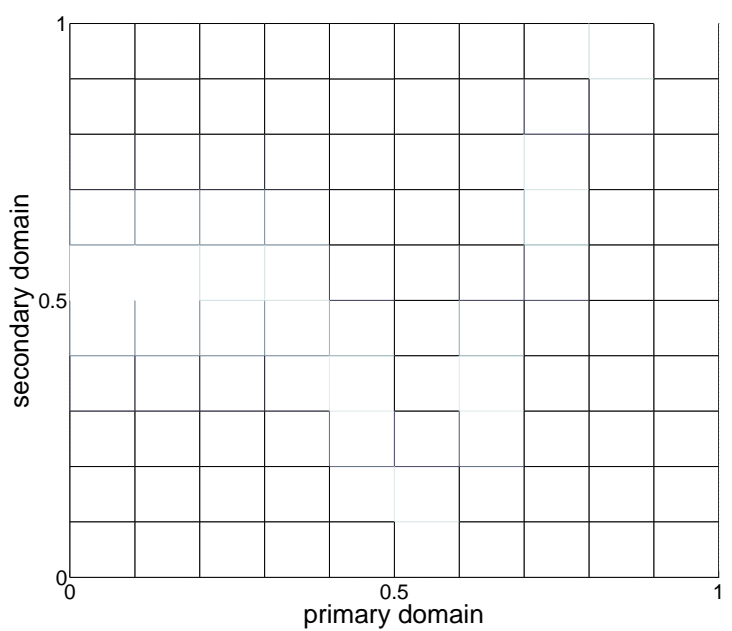

(b) FOU.

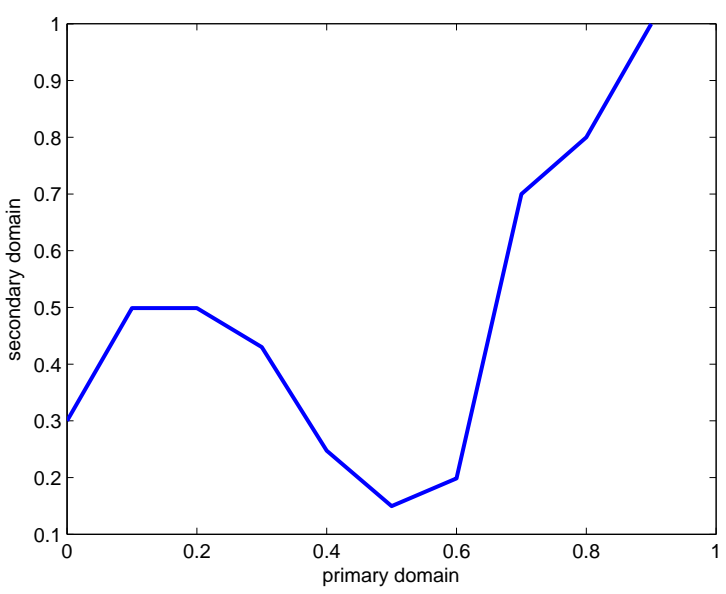

(c) GRESA.

Figure A.10: Shopping0.1 - Shopping FIS generated generalised test set, domain degree of discretisation 0.1. 
[8] L. Dymova, P. Sevastjanov, and A. Tikhonenko. An interval type-2 fuzzy extension of the TOPSIS method using alpha cuts. Knowledge-Based Systems, 83:116 - 127, 2015.

[9] M. El-Bardini and A. M. El-Nagar. Interval type-2 fuzzy PID controller for uncertain nonlinear inverted pendulum system. ISA Transactions, 53(3):732-743, 2014.

[10] Y. Gao, H. Li, L. Wu, H. R. Karimi, and H.-K. Lam. Optimal control of discrete-time interval type-2 fuzzy-model-based systems with D-stability constraint and control saturation. Signal Processing, 120:409-421, 2016.

[11] M. Ghaemi, S. K. Hosseini-Sani, and M. H. Khooban. Direct adaptive general type-2 fuzzy control for a class of uncertain non-linear systems. IET Science, Measurement \& Technology, 8(6):518 $527,2014$.

[12] S. Greenfield. Uncertainty Measurement for the Interval Type-2 Fuzzy Set. pages 183 - 194, Zakopane, Poland, 2016. 15th International Conference on Artificial Intelligence and Soft Computing (ICAISC 2016).

[13] S. Greenfield and F. Chiclana. Combining the $\alpha$-Plane Representation with an Interval Defuzzification Method. In Proceedings of EUSFLAT-LFA 2011, pages 920 - 927, Aix-les-Bains, France, July 2011.

[14] S. Greenfield and F. Chiclana. The Structure of the Type-Reduced Set of a Continuous Type-2 Fuzzy Set. In Proceedings of EUSFLAT 2013 (Advances in Intelligent Systems Research, Atlantis Press), Milan, Italy, September 2013. DOI: http://dx.doi.org/10.2991/eusflat.2013.102.

[15] S. Greenfield and F. Chiclana. Defuzzification of the Discretised Generalised Type-2 Fuzzy Set: Experimental Evaluation. Information Sciences, 244:1 - 25, September 2013. DOI: http://dx.doi.org/10.1016/j.ins.2013.04.032.

[16] S. Greenfield and F. Chiclana. Accuracy and Complexity Evaluation of Defuzzification Strategies for The Discretised Interval Type-2 Fuzzy Set. International Journal of Approximate Reasoning, 54(8):1013 - 1033, October 2013. DOI: http://dx.doi.org/10.1016/j.ijar.2013.04.013.

[17] S. Greenfield and F. Chiclana. Fuzzy in 3D: Two Contrasting Paradigms. Archives for the Philosophy and History of Soft Computing, 2, 2015. http://www.aphsc.org/index.php/aphsc/ article/view/24 [Online; accessed 20-January-2016].

[18] S. Greenfield and F. Chiclana. Slicing Strategies for the Generalised Type-2 Mamdani Fuzzy Inferencing System. pages 195 - 205, Zakopane, Poland, 2016. 15th International Conference on Artificial Intelligence and Soft Computing (ICAISC 2016).

[19] S. Greenfield and R. I. John. The Uncertainty Associated with a Type-2 Fuzzy Set. In R. Seising (editor) Views on Fuzzy Sets and Systems from Different Perspectives, in 'Studies in Fuzziness and Soft Computing', series editor J. Kacprzyk, volume 243, pages 471 - 483. Springer-Verlag, 2009. DOI: http://dx.doi.org/10.1007/978-3-540-93802-6_23.

[20] S. Greenfield, F. Chiclana, S. Coupland, and R. I. John. The Collapsing Method of Defuzzification for Discretised Interval Type-2 Fuzzy Sets. Information Sciences, 179(13):2055 - 2069, June 2009. DOI: http://dx.doi.org/10.1016/j.ins.2008.07.011. 
[21] S. Greenfield, F. Chiclana, and R. I. John. The Collapsing Method: Does the Direction of Collapse Affect Accuracy? In Proceedings of IFSA-EUSFLAT 2009, pages 980 - 985, Lisbon, Portugal, July 2009.

[22] S. Greenfield, F. Chiclana, R. I. John, and S. Coupland. The Sampling Method of Defuzzification for Type-2 Fuzzy Sets: Experimental Evaluation. Information Sciences, 189:77 - 92, April 2012. DOI: http://dx.doi.org/10.1016/j.ins.2011.11.042.

[23] A. Halder, A. Konar, R. Mandal, A. Chakraborty, P. Bhowmik, N. R. Pal, and A. K. Nagar. General and interval type-2 fuzzy face-space approach to emotion recognition. IEEE Transactions on Systems, Man, and Cybernetics: Systems, 43(3):587.

[24] R. I. John and S. Coupland. Type-2 Fuzzy Logic: A Historical View. IEEE Computational Intelligence Magazine, 2(1):57 - 62, February 2007. DOI: 10.1109/MCI.2007.357194.

[25] N. N. Karnik and J. M. Mendel. Centroid of a Type-2 Fuzzy Set. Information Sciences, 132:195 220, 2001.

[26] N. N. Karnik, J. M. Mendel, and Q. Liang. Type-2 Fuzzy Logic Systems. IEEE Transactions on Fuzzy Systems, 7(6):643 - 658, 1999.

[27] T. Kumbasar and H. Hagras. A self-tuning zSlices-based general type-2 fuzzy PI controller. IEEE Transactions on Fuzzy Systems, 23(4):991.

[28] T. Kumbasar and H. Hagras. Big Bang-Big Crunch optimization based interval type-2 fuzzy PID cascade controller design strategy. Information Sciences, 282:277 - 295, 2014.

[29] W. Van Leekwijck and E. E. Kerre. Defuzzification: Criteria and Classification. Fuzzy Sets and Systems, 108:159 - 178, 1999. DOI: 10.1016/j.fss.2008.06.018.

[30] O. Linda and M. Manic. Uncertainty Modelling for Interval Type-2 Fuzzy Logic Systems Based on Sensor Characteristics. In Proceedings of the IEEE Symposium on Advances in Type-2 Fuzzy Logic Systems 2011, Paris, April 2011.

[31] O. Linda and M. Manic. General Type-2 Fuzzy C-Means Algorithm for Uncertain Fuzzy Clustering. IEEE Transactions on Fuzzy Systems, 20(5):883 - 897, October 2012. DOI: 10.1109/TFUZZ.2012.2187453.

[32] F. Liu. An Efficient Centroid Type-Reduction Strategy for General Type-2 Fuzzy Logic System. Information Sciences, 178(9):2224-2236, 2008. DOI: 10.1016/j.ins.2007.11.014.

[33] Y.-X. Liu, J.-S. Shieh, F. Doctor, S.-Z. Fan, and K.-K. Jen. Multivariable type-2 self-organizing fuzzy logic controllers for regulating anesthesia with rule base extraction. In Conference on Technologies and Applications of Artificial Intelligence, pages 217 - 222, 2013.

[34] L. Alberto Lucas, T. Mezzadri Centeno, and M. Regattieri Delgado. General Type-2 Fuzzy Inference Systems: Analysis, Design and Computational Aspects. In Proceedings of FUZZ-IEEE 2007, pages 1743-1747, London, 2007.

[35] G. E. Martínez, O. Mendoza, J. R. Castro, P. Melin, and O. Castillo. Generalized type-2 fuzzy logic in response integration of modular neural networks. In Joint IFSA World Congress and NAFIPS Annual Meeting (IFSA/NAFIPS), page 1331. 
[36] F. Di Martino and S. Sessa. Type-2 interval fuzzy rule-based systems in spatial analysis. Information Sciences, 279:199 - 212, 2014.

[37] P. Melin, C. I. Gonzalez, J. R. Castro, O. Mendoza, and O. Castillo. Edge-detection method for image processing based on generalized type-2 fuzzy logic. IEEE Transactions on Fuzzy Systems, 22(6): $1515-1525,2014$.

[38] J. M. Mendel. Uncertain Rule-Based Fuzzy Logic Systems: Introduction and New Directions. Prentice-Hall PTR, 2001. ISBN 0-13-040969-3.

[39] J. M. Mendel. On KM algorithms for solving type-2 fuzzy set problems. IEEE Transactions on Fuzzy Systems, 21(3):426 - 446, 2013.

[40] J. M. Mendel and R. I. John. Type-2 fuzzy sets made simple. IEEE Transactions on Fuzzy Systems, 10(2):117 - 127, 2002. DOI: http://dx.doi.org/10.1109/91.995115.

[41] J. M. Mendel, F. Liu, and D. Zhai. $\alpha$-Plane Representation for Type-2 Fuzzy Sets: Theory and applications. IEEE Transactions on Fuzzy Systems, 17(5):1189 - 1207, 2009.

[42] J. M. Mendel, M. R. Rajati, and P. Sussner. On clarifying some definitions and notations used for type-2 fuzzy sets as well as some recommended changes. Information Sciences, 340:337 - 345, 2016.

[43] S. Naim and H. Hagras. A general type-2 fuzzy logic based approach for multi-criteria group decision making. In Proceedings of FUZZ-IEEE 2014, page 1.

[44] T. Nguyen, A. Khosravi, D. Creighton, and S. Nahavandi. Medical data classification using interval type-2 fuzzy logic system and wavelets. Applied Soft Computing, 30:812 - 822, 2015.

[45] M. Nie and W. W. Tan. Towards an Efficient Type-Reduction Method for Interval Type-2 Fuzzy Logic Systems. In Proceedings of FUZZ-IEEE 2008, pages 1425 - 1432, Hong Kong, June 2008.

[46] M. A. Sanchez, O. Castillo, and J. R. Castro. Generalized type-2 fuzzy systems for controlling a mobile robot and a performance comparison with interval type-2 and type-1 fuzzy systems. Expert Systems with Applications, 42(14):5904 - 5914, 2015.

[47] R. Shahnazi. Observer-based adaptive interval type-2 fuzzy control of uncertain MIMO nonlinear systems with unknown asymmetric saturation actuators. Neurocomputing, 171:1053 - 1065, 2016.

[48] A. D. Torshizi, L. Petzold, and M. Cohen. Direct higher order fuzzy rule-based classification system: Application in mortality prediction. In IEEE International Conference on Bioinformatics and Biomedicine (BIBM), page 846.

[49] C. Wagner and H. Hagras. Toward General Type-2 Fuzzy Logic Systems based on zSlices. IEEE Transactions on Fuzzy Systems, 18(4):637 - 660, 2010.

[50] D. Wu. Approaches for reducing the computational cost of interval type-2 fuzzy logic systems: overview and comparisons. IEEE Transactions on Fuzzy Systems, 21(1):80 - 99, 2013.

[51] D. Wu and J. M. Mendel. Enhanced Karnik-Mendel Algorithms. IEEE Transactions on Fuzzy Systems, 17(4):923 - 934, August 2009. 
[52] E. Yesil. Interval type-2 fuzzy PID load frequency controller using Big Bang-Big Crunch optimization. Applied Soft Computing, 15:100 - 112, 2014.

[53] L. A. Zadeh. Fuzzy Sets. Information and Control, 8:338 - 353, 1965.

[54] L. A. Zadeh. The Concept of a Linguistic Variable and its Application to Approximate Reasoning. Information Sciences, 8:199 - 249, 1975.

[55] S.-M. Zhou, F. Chiclana, R. I. John, and J. M. Garibaldi. Type-1 OWA Operators for Aggregating Uncertain Information with Uncertain Weights Induced By Type-2 Linguistic Quantifiers. Fuzzy Sets and Systems (ISSN 0165-0114), 159(24):3281 - 3296, December 2008. DOI: http://dx.doi.org/10.1016/j.fss.2008.06.018. 